\title{
Two-dimensional Radiative Magnetohydrodynamic Simulations of Partial Ionization in the Chromosphere. II. Dynamics and Energetics of the Low Solar Atmosphere
}

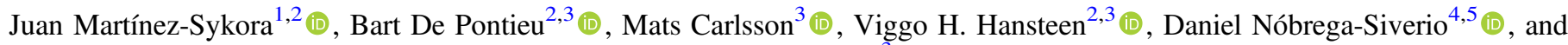 \\ Boris V. Gudiksen ${ }^{3}$ (iD \\ ${ }^{1}$ Bay Area Environmental Research Institute, Petaluma, CA 94952, USA; juanms@1msal.com \\ ${ }^{2}$ Lockheed Martin Solar and Astrophysics Laboratory, Palo Alto, CA 94304, USA \\ ${ }^{3}$ Institute of Theoretical Astrophysics, University of Oslo, P.O. Box 1029 Blindern, NO-0315 Oslo, Norway \\ ${ }^{4}$ Instituto de Astrofísica de Canarias, E-38200 La Laguna (Tenerife), Spain \\ ${ }^{5}$ Department of Astrophysics, Universidad de La Laguna, E-38200 La Laguna (Tenerife), Spain \\ Received 2017 June 8; revised 2017 August 15; accepted 2017 August 22; published 2017 September 20
}

\begin{abstract}
We investigate the effects of interactions between ions and neutrals on the chromosphere and overlying corona using 2.5D radiative MHD simulations with the Bifrost code. We have extended the code capabilities implementing ion-neutral interaction effects using the generalized Ohm's law, i.e., we include the Hall term and the ambipolar diffusion (Pedersen dissipation) in the induction equation. Our models span from the upper convection zone to the corona, with the photosphere, chromosphere, and transition region partially ionized. Our simulations reveal that the interactions between ionized particles and neutral particles have important consequences for the magnetothermodynamics of these modeled layers: (1) ambipolar diffusion increases the temperature in the chromosphere; (2) sporadically the horizontal magnetic field in the photosphere is diffused into the chromosphere, due to the large ambipolar diffusion; (3) ambipolar diffusion concentrates electrical currents, leading to more violent jets and reconnection processes, resulting in (3a) the formation of longer and faster spicules, (3b) heating of plasma during the spicule evolution, and (3c) decoupling of the plasma and magnetic field in spicules. Our results indicate that ambipolar diffusion is a critical ingredient for understanding the magnetothermodynamic properties in the chromosphere and transition region. The numerical simulations have been made publicly available, similar to previous Bifrost simulations. This will allow the community to study realistic numerical simulations with a wider range of magnetic field configurations and physics modules than previously possible.
\end{abstract}

Key words: magnetohydrodynamics (MHD) - methods: numerical - radiative transfer - Sun: atmosphere - Sun: chromosphere - Sun: corona

Supporting material: animations

\section{Introduction}

The chromosphere (the interface between the photosphere and the million-degree corona) is of great interest because it processes enough nonthermal energy to heat the entire corona, and all nonthermal energy that powers the corona and solar wind passes first through the chromosphere. The chromosphere thus plays a key role in the energy and mass balance of the outer solar atmosphere (Athay \& Holzer 1982; Dere et al. 1989; De Pontieu et al. 2011) and is at the root of the solar wind (Withbroe \& Noyes 1977; De Pontieu et al. 2007b; Tomczyk et al. 2007; McIntosh et al. 2011). However, it is a very complex region that is difficult to directly diagnose. This is because many complex physical processes play a role in the chromosphere: (1) the plasma is not in local thermodynamic equilibrium (LTE); (2) the radiation is optically thick; (3) the radiation suffers scattering; (4) the ionization is not in equilibrium; (5) the gas is partially ionized; (6) in the upper chromosphere, transition region (TR), and corona, thermal conduction plays an important role in transporting energy along the magnetic field. In addition, the chromosphere is also distinguished by several transitions: from gas pressure dominated to magnetic field dominated, from collisional to collisionless, and from partial to full ionization. Note that many of the physical processes (especially 1-4) imply that the interpretation of imaging and spectral observations is often difficult and requires modeling.
The chromosphere is highly dynamic: it is permeated by upward-traveling shocks (Hansteen et al. 2006; De Pontieu et al. 2007a), and its interface with the TR and corona is dominated by short-lived jets or spicules. Many phenomena in the chromosphere remain poorly understood. For example, it is not clear how the chromosphere is heated in both nonmagnetic regions (Carlsson et al. 2007) and magnetic regions (Carlsson et al. 2015). It also remains unclear how small-scale flux emerges in the chromosphere: the chromosphere appears to be filled with magnetic field, even in quiet Sun, despite the fact that the photosphere is subadiabatic, which should, in principle, thwart the expansion of emerging magnetic flux into the corona (Acheson 1979; Archontis et al. 2004). The formation of spicules has similarly remained mysterious with a multitude of models proposed (Sterling et al. 2010; Tsiropoula et al. 2012), but most failing to reproduce the properties of spicules as they are now measured with high-resolution instruments (De Pontieu et al. 2007a; Pereira et al. 2012); for example, these models fail to reproduce the very high speeds $\left(50 \mathrm{~km} \mathrm{~s}^{-1}\right.$ or more), the temperature evolution of observed chromospheric spicules or the short-lived rapid blue events seen in the spectral profiles of Ca II or $\mathrm{H} \alpha$ (Martínez-Sykora et al. 2013), or the heating to TR temperatures. Recently, Martiunez-Sykora et al. (2017a) used the same model as the current paper to propose a new spicule formation mechanism that can reproduce the observed properties of spicules. This model has the potential to resolve some of the remaining unresolved issues with respect to 
the impact of spicules on the TR and corona (De Pontieu et al. 2011; Madjarska et al. 2011; Judge et al. 2012). Other phenomena are better understood, but current models cannot fully capture some of their properties, e.g., the length and lifetime of dynamic fibrils (Suematsu et al. 1995; Hansteen et al. 2006; Heggland et al. 2007; Martínez-Sykora et al. 2009b; Iijima \& Yokoyama 2015). The persistence of these unresolved issues in part stems from the difficulty of combining all these ingredients into a single numerical approach that models the full solar atmosphere and takes into account radiative transfer with scattering, thermal conduction, partial ionization effects, etc.

Significant efforts have focused on combining 3D MHD equations with radiative transfer and thermal conduction along the magnetic field (Stein \& Nordlund 2006; Abbett 2007; Gudiksen et al. 2011; Bingert \& Peter 2011; Rempel 2017). Furthermore, recently Leenaarts et al. (2007) and Golding et al. (2014) expanded the Bifrost code to include nonequilibrium ionization. Cheung \& Cameron (2012) implemented the Hall term in photospheric simulations of the $3 \mathrm{D}$ radiative MHD MURAM code. In this paper we use a version of the Bifrost code that includes the Hall term and Pedersen dissipation in an atmosphere that spans from the convection zone to the lower corona. Our first results (Martínez-Sykora et al. 2012) focused on the validity of the generalized Ohm's law (GOL) and on the spatiotemporal properties of the Hall term and the ambipolar diffusion in a $2 \mathrm{D}$ radiative MHD solar atmosphere. Here we will focus on the impact of these terms on the magnetothermodynamic properties of the solar atmosphere.

In a magnetized partially ionized gas, ions are coupled to the magnetic field, whereas neutrals are not directly affected by the magnetic field and, in principle, can move "freely." This can lead to a velocity drift between ions and neutrals; consequently, the bulk motion of the combined fluid can, under certain circumstances, be different from the motion of the magnetic flux (Martínez-Sykora et al. 2016). Sufficient collisions between ions and neutrals can couple the neutrals to the magnetic field, while at the same time to some extent decouple the ions from the magnetic field. Under certain conditions, i.e., when collisions are sufficient to ensure equal temperatures of the ions, neutrals, and electrons, and in addition that timescales are greater than the ion-neutral collision timescales, one can still solve the single-fluid MHD equations and expand Ohm's law in order to include the ion-neutral interaction effects, by adding the so-called Hall term and ambipolar diffusion (see Cowling 1957; Braginskii 1965; Parker 2007, among others). As a result of ambipolar diffusion, the magnetic field can diffuse and magnetic energy will be dissipated and lead to heating. These are the effects implemented in the $3 \mathrm{D}$ radiative MHD Bifrost code (Section 2).

During the past two decades, significant progress has been made in understanding the potential impact of partial ionization in the lower solar atmosphere, mostly using more idealized models. Partial ionization in the chromosphere is, for instance, known to lead to dissipation of Alfvénic waves through ionneutral collisions (De Pontieu \& Haerendel 1998; De Pontieu 1999; De Pontieu et al. 2001; Leake et al. 2005; Forteza et al. 2007; Soler et al. 2009, 2012, 2013, 2015, among others). However, the picture is far from complete since it remains unknown whether this dissipation plays a significant role in heating the chromosphere: previous work was based on highly idealized models (usually assuming a static or structureless chromosphere) and most often used fixed values for ion-neutral collision frequencies, ignoring the intricate dynamic balance between heating, ionization, and cooling that continuously takes place in the chromosphere. Previous work has also found that electrical currents perpendicular to the magnetic field can be dissipated by Pedersen dissipation and lead to heating (e.g., Arber et al. 2009; Goodman \& Judge 2012; Khomenko \& Collados 2012). Pedersen dissipation also allows emerging magnetic field to diffuse more rapidly into the atmosphere (Leake \& Arber 2006; Arber et al. 2007; Leake \& Linton 2013). Compared to single-fluid simulations, 2.5D simulations of prominences including partial ionization show an increase of small-scale velocities as a result of the nonlinearity of the Rayleigh-Taylor instability (Díaz et al. 2014; Khomenko et al. 2014). Further details of such processes can be found in the review by Leake et al. (2014), which details the impact of ion-neutral collisions and their properties in the solar chromosphere and Earth's ionosphere. Martínez-Sykora et al. (2015) summarize and discuss the role of ion-neutral interaction effects in the solar atmosphere.

This paper is structured as follows: In Section 2 we briefly describe the Bifrost code (Gudiksen et al. 2011), as well as the numerical methods used to model the ion-neutral interaction effects. We study the impact of ion-neutral effects by studying two simulations, one without and one with ion-neutral interaction effects. The initial conditions and the setup of the two simulations are detailed in Section 3. In the Appendix we provide details on the publicly available snapshots from the simulations. We analyze these models in detail in Section 4, where we focus on general aspects of the thermodynamic properties (Section 4.1), the magnetic field distribution (Section 4.2), the heating (Section 4.3), and the transport of magnetic flux (Section 4.4). We continue with a description of three representative processes that we consider of great interest (Section 4.5): expanding cold chromospheric bubbles (Section 4.5.1), chromospheric jets (Section 4.5.2), and chromospheric reconnection in regions with highly inclined field (Section 4.5.3). We finish the paper with a discussion and conclusions in Section 5.

\section{Equations and Numerical Method}

The photosphere, chromosphere, and TR are partially ionized, and the interaction between ionized and neutral particles has important consequences. We investigate this physical process by modeling the solar atmosphere with the Bifrost code. This code solves the full MHD equations with radiative transfer and thermal conduction along the magnetic field. The numerical methods implemented in the code have been described in detail by Gudiksen et al. (2011). In addition, the modules for the optically thick radiation and the numerical recipes for the radiative transfer in the chromosphere and TR are described by Hayek et al. (2010) and Carlsson \& Leenaarts (2012), respectively. In order to implement the ion-neutral interaction effects, we take into account that the code explicitly solves the MHD equations on a Cartesian staggered mesh. In order to suppress numerical noise, high-order artificial diffusion is added in the form of both viscosity and magnetic diffusivity (see Galsgaard \& Nordlund 1995; Martínez-Sykora et al. 2009a; Gudiksen et al. 2011).

We implemented partial ionization effects in the Bifrost code by adding two new terms in the induction equation, i.e., the 
Hall term and the ambipolar diffusion:

$$
\begin{aligned}
\frac{\partial \boldsymbol{B}}{\partial t}= & \nabla \times\left[\boldsymbol{u} \times \boldsymbol{B}-\eta \boldsymbol{J}-\frac{\eta_{\text {hall }}}{|B|} \boldsymbol{J} \times \boldsymbol{B}\right. \\
& \left.+\frac{\eta_{\text {amb }}}{B^{2}}(\boldsymbol{J} \times \boldsymbol{B}) \times \boldsymbol{B}\right],
\end{aligned}
$$

where $\boldsymbol{B}, \boldsymbol{J}, \boldsymbol{u}$, and $\eta$ are magnetic field, current density, velocity, and ohmic diffusion, respectively (see Cowling 1957; Braginskii 1965; Parker 2007; Pandey \& Wardle 2008; Martínez-Sykora et al. 2012; Leake et al. 2014, for the derivation of this equation). The Bifrost code does not calculate the Spitzer ohmic dissipation since the artificial diffusion plays this role and is much larger than the Spitzer ohmic diffusion. The $\eta_{\text {hall }}$ term and the $\eta_{\text {amb }}$ diffusive term are

$$
\begin{gathered}
\eta_{\text {hall }}=\frac{|B|}{q_{e} n_{e}}, \\
\eta_{\mathrm{amb}}=\frac{\left(|B| \rho_{n} / \rho\right)^{2}}{\rho_{i} \nu_{\text {in }}}=\frac{\left(|B| \rho_{n} / \rho\right)^{2}}{\rho_{n} \nu_{n i}},
\end{gathered}
$$

where $\rho_{i}, \rho_{n}, \rho, \nu_{i n}, \nu_{n i}, n_{e}$, and $q_{e}$ are ion mass density, neutral mass density, total mass density, ion-neutral collision frequency, neutral-ion collision frequency, electron number density, and the absolute value of the electron charge, respectively. Note that $\eta_{\text {hall }}$ is not a diffusive or dissipative term, in contrast to $\eta$ and $\eta_{\mathrm{amb}}$. The ambipolar diffusion should not be confused with the so-called ambipolar drift used in plasma physics. Ambipolar diffusion is also referred to as Pedersen dissipation. Since we are using the mathematical expression of the ambipolar diffusion (see Parker 2007) instead of the Pedersen dissipation (compare with Equation (46) in Leake et al. 2014) in the code, we refer to this physical process as ambipolar diffusion.

We implement four different approximations to compute the ion-neutral collision frequency. Three of them have been already discussed in Martínez-Sykora et al. (2012): one approximation follows Osterbrock (1961) and De Pontieu \& Haerendel (1998), the second one follows von Steiger \& Geiss (1989), and the third one follows Fontenla et al. (1993). The fourth approximation to compute the ion-neutral collision frequency is using recent studies that improve the estimation of the collisional cross sections under chromospheric conditions (Vranjes \& Krstic 2013). These various approximations for the calculation of the collision frequency between ions and neutrals lead to large differences in the values of ambipolar diffusion as shown by Martínez-Sykora et al. (2012). These differences also lead to different thermal properties of the solar atmosphere (Martínez-Sykora et al. 2015). Therefore, it is critical to properly calculate the ambipolar diffusion. As a result, we present here only results based on the most recent and state-ofthe-art approximation of the ion-neutral cross sections described by Vranjes \& Krstic (2013). For this, we take into account the temperature dependence of the cross sections for $\mathrm{p}-$ $\mathrm{H}$, p-He, and $\mathrm{He}^{+}-\mathrm{He}$ collisions. The cross sections are calculated by combining quantum and classical theory. In addition, we consider the 16 most important elements. The collisional cross sections of these elements are not well known. As an approximation and following Vranjes et al. (2008), the cross section between any other element and neutrals is chosen to be the value of the cross section for protons multiplied by $m_{m} / m_{p}$, where $m_{m}$ is the atomic mass of the considered element and $m_{p}$ is the proton mass.

To solve the Hall term and the ambipolar diffusion when evaluating the partial differential equations, we apply a method similar to that used in the Bifrost code, i.e., a sixth-orderaccurate operator for determining the partial spatial derivatives.

Since we can reformulate Equation (1) as

$$
\frac{\partial \boldsymbol{B}}{\partial t}=\nabla \times\left[\boldsymbol{u} \times \boldsymbol{B}-\eta \boldsymbol{J}-\boldsymbol{u}_{H} \times \boldsymbol{B}+\boldsymbol{u}_{A} \times \boldsymbol{B}\right],
$$

where the "Hall velocity" is $\boldsymbol{u}_{H}=\left(\eta_{\text {hall }} \boldsymbol{J}\right) /|B|$ and the "ambipolar velocity" is $\boldsymbol{u}_{A}=\left(\eta_{\mathrm{amb}} \boldsymbol{J} \times \boldsymbol{B}\right) / B^{2}$, the Hall term and ambipolar diffusivity impose two new constraints on the Courant Friedrichs and Lewy (CFL) condition (Courant et al. 1928), which restrict the time interval between numerical steps $\left(\Delta t_{H}=\Delta x / \boldsymbol{u}_{H}\right.$ and $\left.\Delta t_{A}=\Delta x / \boldsymbol{u}_{A}\right)$. Both velocities are a function of the current $(\nabla \times \boldsymbol{B})$, i.e., both CFL conditions are quadratic functions in $\Delta x$, and the time step will therefore decrease quadratically with increasing spatial resolution (Cheung \& Cameron 2012). In addition to these CFL conditions, the whistler phase speed $\left(u_{w}=k u_{a}^{2} / \Omega_{i}\right.$, where $u_{a}$ is the Alfvén velocity, $\Omega_{i}$ is the ion cyclotron velocity, and $k$ is the wavenumber) is also required for completeness of the CFL condition for the Hall term (Huba 2003).

These new CFL restrictions only apply to the induction equation, which contains the Hall and ambipolar terms. Therefore, we solve the induction equation on a separate timescale, $N_{\mathrm{Ist}}=\Delta t_{\mathrm{MHD}} / \Delta t_{\mathrm{GOL}}$ times for each time that the MHD equations are solved, where $\Delta t_{\mathrm{MHD}}$ is the smallest time step interval due to the classical CFL condition on the MHD equations and $\Delta t_{\mathrm{GOL}}=\min \left(\Delta t_{H}, \Delta t_{A}\right)$ is the time step for the induction equation (i.e., following a similar approach to that of Leake \& Arber 2006). The result from solving the induction equation $N_{\text {Ist }}$ times feeds the MHD equations with an updated magnetic field and Joule heating coming from the artificial and ambipolar dissipation. If during the $N_{\text {Ist }}$ iterations the ratio between the internal energy and the accumulative magnetic energy release is larger than a certain threshold $\left(e / Q_{\text {Joule }}>\Delta t_{\mathrm{MHD}}\right)$, we allow the iterations to be interrupted and the MHD equations to be solved again. This constraint is based on the magnitude of the magnetic energy release from the ambipolar diffusion. It can be calculated accurately since the induction equation advances in time explicitly and the accumulated dissipated magnetic energy is calculated for each small time step.

Numerical errors coming from the two new terms in the induction equation are diffused away using hyperdiffusive operators. Taking into account Equation (4), we add a hyperdiffusive term similar to that described in Gudiksen et al. (2011) (and previously Galsgaard \& Nordlund 1995) to the advection term $(\nabla \times \boldsymbol{u} \times \boldsymbol{B})$ in the induction equation

$$
\begin{aligned}
\frac{\partial \boldsymbol{B}}{\partial t}= & \ldots+\frac{\partial}{\partial x}\left\{\left[\max \left(\nu_{h 1}\left|\boldsymbol{u}_{H}\right|, \nu_{a 1}\left|\boldsymbol{u}_{A}\right|\right)\right.\right. \\
& \left.\left.+\max \left(\nu_{h 2} \Delta x \nabla_{x}^{1} u_{H x}, \nu_{a 2} \Delta x \nabla_{x}^{1} u_{A x}\right)\right] \frac{\partial \boldsymbol{B}}{\partial x} Q\left(\frac{\partial \boldsymbol{B}}{\partial x}\right)\right\},
\end{aligned}
$$




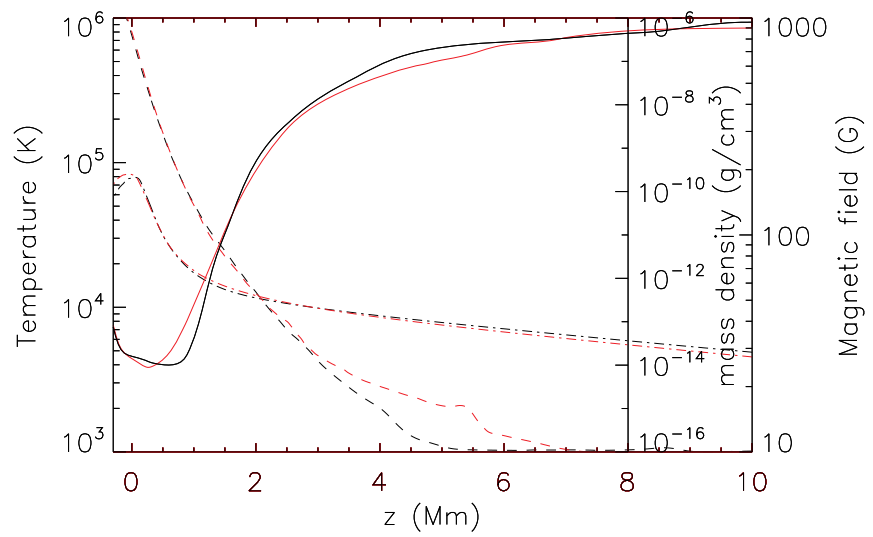

Figure 1. Horizontal and time averages for the temperature (solid line), unsigned magnetic field (dot-dashed line), and mass density (dashed line) for the non-GOL (black) and GOL (red) simulations as a function of height integrated between snapshots 300 and 370, i.e., over 11 minutes.

where $\nabla_{x}^{1}$ is the first-order gradient in the $x$ direction and $\nu_{a 1}$, $\nu_{a 2}, \nu_{h 1}$, and $\nu_{h 2}$ are constant parameters of order $10^{-2}$. Finally,

$$
Q(g)=\frac{\left|\frac{\partial^{2} g}{\partial x^{2}}\right|}{|g|+\frac{1}{q}\left|\frac{\partial^{2} g}{\partial x^{2}}\right|},
$$

with $q$ the quench number, a constant of order 10, and $g$ a function. An extra hyperdiffusive term is necessary for removing numerical errors coming from the ambipolar diffusion where $\eta_{\mathrm{amb}}$ is high and the current is low:

$$
\frac{\partial \boldsymbol{B}}{\partial t}=\ldots+\frac{\partial}{\partial x}\left\{\nu \Delta x\left[\frac{\nu_{a 3} \nabla_{x}^{1} u_{A x}}{|\boldsymbol{J} \boldsymbol{\Delta} \boldsymbol{x}| /|\boldsymbol{B}|}\right] \frac{\partial \boldsymbol{B}}{\partial x} Q\left(\frac{\partial \boldsymbol{B}}{\partial x}\right)\right\},
$$

where $\nu_{a 3}$ is a constant parameter of order $10^{-2}$ and $J \Delta x_{x}=(\partial B z / \partial y) \Delta y-(\partial B y / \partial z) \Delta z$. This hyperdiffusive splitting of the diffusive terms into local and global components makes it possible to run the code with a global diffusivity that is at least a factor of 10 less than if the global term were the only one implemented in the code. This implementation has been tested in Martínez-Sykora et al. (2012).

All the diffused magnetic energy coming from the high-order artificial hyperdiffusion and the ambipolar diffusion is converted into thermal energy by Joule heating given by $Q_{\text {Joule }}=\boldsymbol{E} \cdot \boldsymbol{J}$, where the electric field $\boldsymbol{E}$ is calculated from the current $\boldsymbol{J}$.

\section{Models and Initial Conditions}

The study presented here is focused on two different $2.5 \mathrm{D}$ models computed using the Bifrost code. Both models are calculated for a numerical domain that spans from the upper layers of the convection zone ( $3 \mathrm{Mm}$ below the photosphere) to the corona ( $40 \mathrm{Mm}$ above the photosphere). Convective motions perform work on the magnetic field and introduce magnetic field stresses in the corona. This energy is dissipated and creates the corona self-consistently as the energy deposited by Joule heating is spread through thermal conduction (Gudiksen \& Nordlund 2002), and the temperature becomes on average about a million degrees (see solid lines in Figure 1). Figure 1 shows the horizontal and time (integrated over 11 minutes) averages for the temperature, unsigned magnetic field, and mass density as a function of height. The horizontal domain spans $96 \mathrm{Mm}$. The spatial resolution is uniform along the horizontal axis $(14 \mathrm{~km})$ and nonuniform in the vertical axis, allowing smaller grid size where needed in certain locations, i.e., the resolution from the photosphere (which has an effective temperature of $5800 \mathrm{~K})$ to above the TR $(z=7 \mathrm{Mm})$ is $\sim 12 \mathrm{~km}$, while the grid spacing smoothly increases from the photosphere to the deeper layers of the convection zone and from $z=7 \mathrm{Mm}$ to greater heights up to $\sim 50 \mathrm{~km}$ resolution.

The initial magnetic field has two medium-size plage regions of opposite polarity that are connected and form loops that are up to $\sim 50 \mathrm{Mm}$ long (see Figure 2). The mean unsigned field in the photosphere is $\sim 190 \mathrm{G}$ (dot-dashed line in Figure 1). The initial magnetic field is a potential field. First, we run this setup without ion-neutral interaction effects for roughly 35 minutes after transients have passed through the domain (snapshot 200, $t=0 \mathrm{~s}$ ). From this final instant, we run two simulations: one simulation incorporates the GOL i.e., includes the ion-neutral interaction effects (from now on we will refer to this effect and the model as GOL), and the other is without (non-GOL). Each simulation was run for another $\sim 30$ minutes after transients have disappeared.

\section{Results}

The ion-neutral interactions described above, and implemented through the GOL, strongly influence the state of the simulated chromosphere. In the incoming sections, we will first focus on the differences found in the statistical properties of the models with and without GOL, e.g., the temperature, density, kinetic energy, and magnetic field distribution. Following this, we will reveal the physical mechanisms that are the root causes behind these statistical differences. Finally, we will analyze specific chromospheric processes, such as the expanding cold bubbles, jets, and reconnection, and consider the role of the GOL in these.

\subsection{Thermodynamic Properties}

The GOL simulation shows clear differences in thermodynamic properties compared to the non-GOL simulation. This can be seen by considering the joint probability distribution functions (JPDFs) of the density and temperature, using data integrated over 11 minutes and shown in Figure 3. We call particular attention to cold, $T<4000 \mathrm{~K}(\log T<3.64)$, tenuous, $\rho<3.2 \times 10^{-10} \mathrm{~g} \mathrm{~cm}^{-3}(\log \rho<-9.5)$, plasma. This is gas located in the wake of shocks, "cold chromospheric bubbles," which represent the lowest-temperature regions of the chromosphere (Leenaarts et al. 2011), as long as there is no large-scale flux emergence (Martínez-Sykora et al. 2008; Tortosa-Andreu \& Moreno-Insertis 2009; Ortiz et al. 2014). When ion-neutral interaction effects are not included, we find that the temperature in the wake of shocks is much lower than in the GOL model. In fact, an ad hoc heating term is introduced in order to avoid plasma temperatures below $2000 \mathrm{~K}$, which are outside the validity range of the equation of state. While this ad hoc heating term is crucial for the non-GOL simulation, it is rarely necessary in the GOL simulation since the cold chromospheric bubbles remain warm enough to prevent the ad hoc heating term from becoming active.

The coolest regions in the GOL simulation, for densities below $10^{-11} \mathrm{~g} \mathrm{~cm}^{-3}$, are several hundreds of degrees hotter 


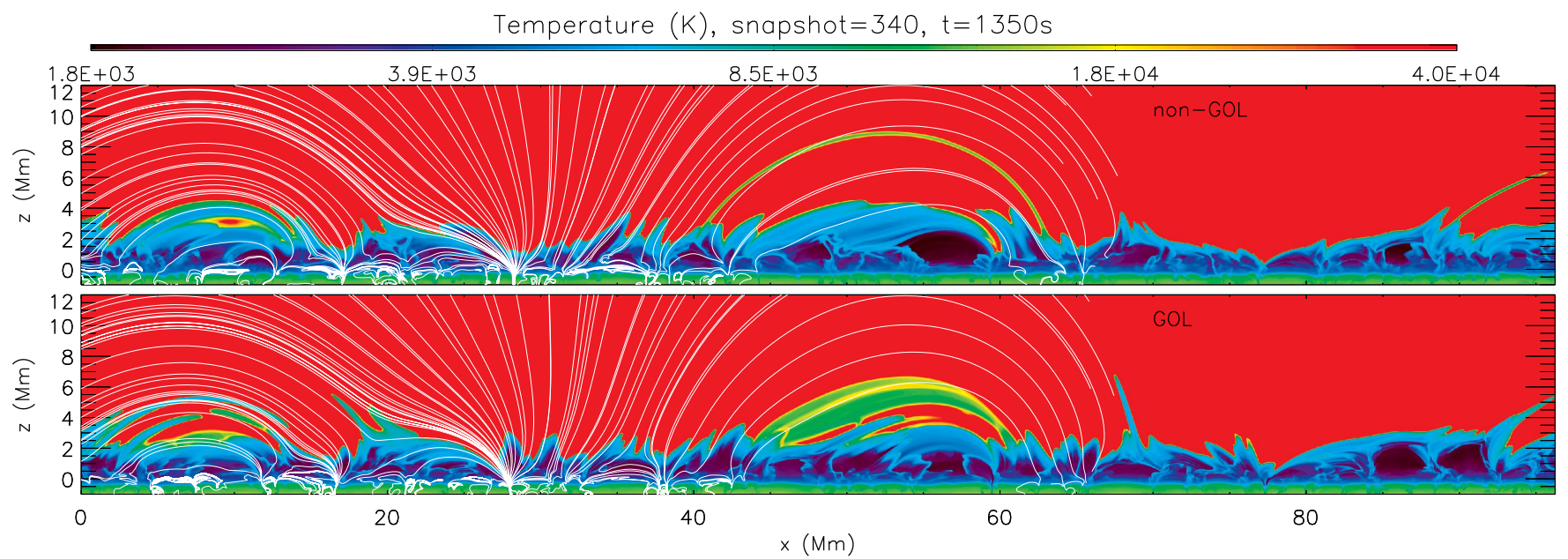

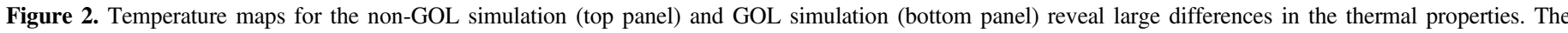

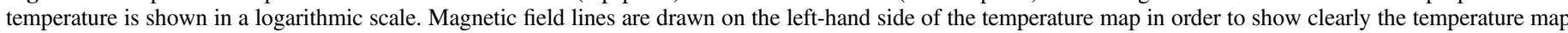
on the right-hand side.

than those in the non-GOL simulation (for which case artificial and ad hoc heating dominates those locations). This results from being heated by the ambipolar diffusion when the cold bubbles reach low densities owing to the expansion. In particular, the large number of neutrals and low ion-neutral collision frequencies in the cool bubbles cause significant dissipation of current perpendicular to the magnetic field (see Sections 4.3 and 4.5.1). This finding, i.e., that the chromosphere remains relatively warm in post-shock wakes, is compatible with observations of the molecular lines of $\mathrm{CO}$ (Penn et al. 2011), which do not reveal absorption everywhere in the quiescent chromosphere. We speculate that the Joule heating contribution from ambipolar diffusion is large enough to prevent the low temperatures necessary for the formation of significant molecular line emission throughout the chromosphere. However, as a caveat, we also note that our simulations are representative for plage regions with rather strong magnetic field. A firm conclusion on this issue will have to wait for simulations that include magnetic field configurations that are typical of quiet Sun.

In contrast to the tenuous cold bubbles, we find that in denser regions, close to the photosphere, the GOL simulation reaches cooler temperatures than the non-GOL simulation. This can be seen in the JPDF (Figure 3) at temperatures below $4000 \mathrm{~K}$ $(\log T<3.6)$ and densities above $10^{-10} \mathrm{~g} \mathrm{~cm}^{-3}$ $(\log \rho>-10)$. This corresponds to a very small fraction $\left(10^{-4}-10^{-5}\right)$ of the upper photosphere and lower chromosphere (dark blue in the color table). The blue area in the $-10<\log \rho<-7$ region of the GOL JPDF (bottom panel) represents regions in which significant quantities of magnetic flux have accumulated in the photosphere. Close to the upper photosphere, the ambipolar diffusion becomes large enough to allow magnetic flux to expand through the photosphere and push material into the upper layers. As this rising magnetic flux expands, it produces regions that are even cooler than those found in the expanding cold bubbles driven by magnetoacoustic shocks (Martínez-Sykora et al. 2008; Tortosa-Andreu \& Moreno-Insertis 2009; Ortiz et al. 2014). This expansion of magnetic flux mediated by ambipolar diffusion does not happen uniformly over the full numerical domain, but only sporadically, in a few locations where both the photospheric magnetic flux and the ambipolar diffusion are large enough (see Sections 4.5.2 and 4.5.3).

We find that the upper chromosphere and TR are more extended in the GOL simulation ( 1.3 times) than the nonGOL by comparing the JPDFs. There is an increase of plasma at temperatures between $5000 \mathrm{~K}(\log T>3.7)$ and $10^{5} \mathrm{~K}$ $(\log T<5)$ with densities between $10^{-11}$ and $10^{-15} \mathrm{~g} \mathrm{~cm}^{-3}$. The spatiotemporal variation of the density is also greater in the upper chromosphere and TR: the density in the GOL simulation reaches both higher and lower densities. Similarly, the corona in the GOL simulation shows a wider range of densities (see the following sections for an explanation).

Finally, the simulations also differ in terms of dynamics. Figure 4 shows the median (left panels) and standard deviation (right panels) of the kinetic energy (averaged over 10 minutes) for the non-GOL (top panel) and GOL (bottom panel) simulations. The middle and upper chromospheres, as well as the TR, contain more kinetic energy and show a larger range of values in the GOL simulation. This is due to the stronger flows and other violent processes happening within the chromosphere (see Sections 4.5.2 and 4.5.3). The enhancements of the kinetic energy and its range of values in the GOL simulation are located in regions where fast chromospheric jets or spicules occur or where reconnection or strong currents are most likely to occur, i.e., $x=[0-24] \mathrm{Mm}, x=[38-70] \mathrm{Mm}$, and $x=[80-96] \mathrm{Mm}$ (see also Figure 2, Sections 4.5.2 and 4.5.3). In this simulation, some regions with open field lines in the corona $(x=30 \mathrm{Mm})$ show enhancements of the kinetic energy, which are caused by an artifact from the open boundary conditions at the top of the numerical domain. These can heat the open field lines under some conditions. The field lines associated with this artifact are localized to a small chromospheric region dominated by magnetoacoustic shocks. The artifact does not impact the lower chromosphere since the magnetoacoustic shocks are similar in nature to those in other open field regions that are unaffected by the artifact.

\subsection{Energy Distribution and Field Topology}

The magnetic field distribution and configuration are clearly different in the two models. Figure 5 shows, for both models, the magnetic free energy normalized by the magnetic energy of 

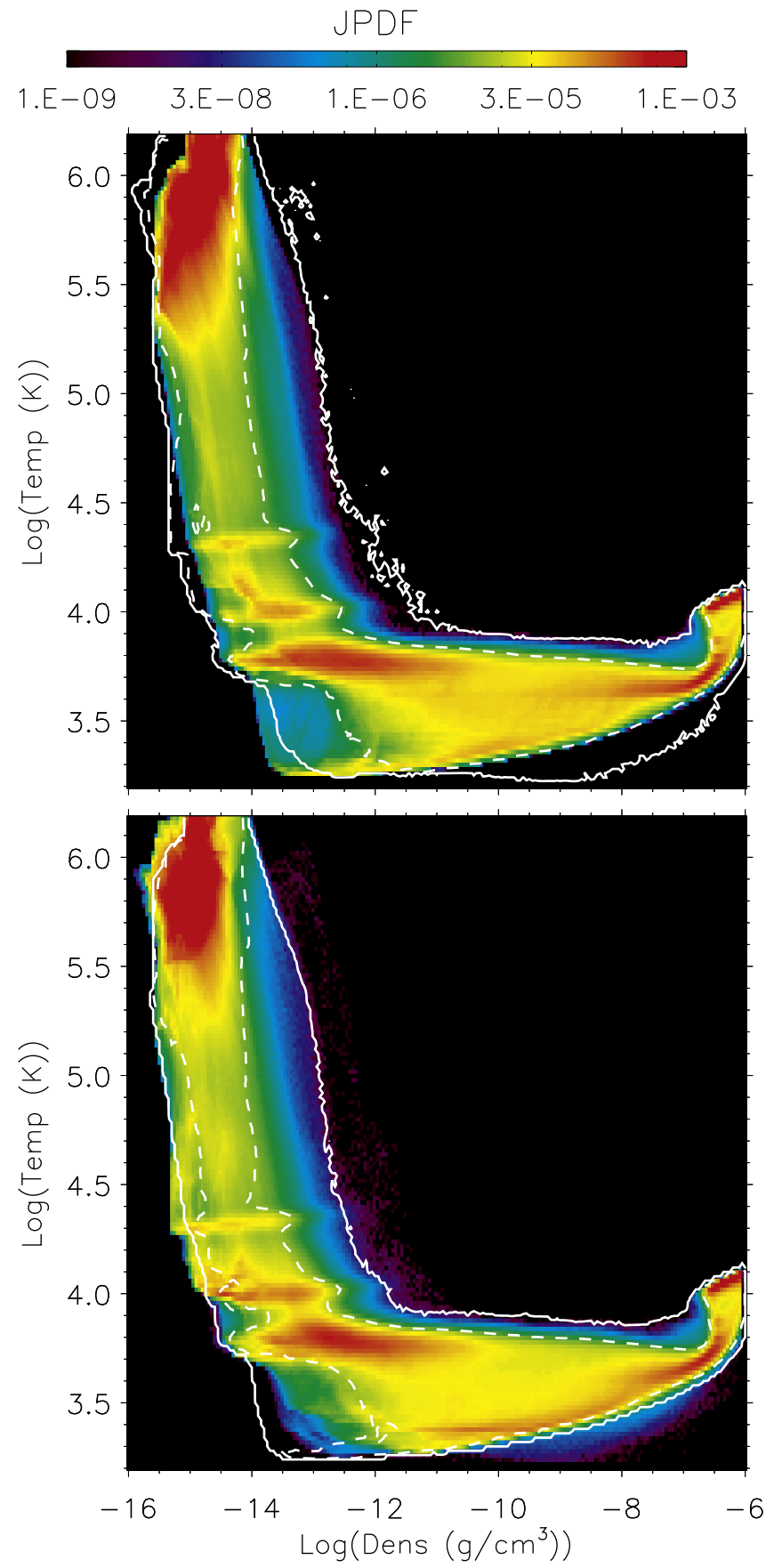

Figure 3. JPDF of temperature (vertical axis) and density (horizontal axis) over 11 minutes (between snapshots 300 and 370) for non-GOL (top) and GOL (bottom) simulations. The white contours correspond to the temperature and density regime of the whole simulation (solid) and at JPDF $=5 \times 10^{-5}$ (dashed) for non-GOL (bottom, whereas the color map corresponds to the simulation GOL) and GOL (top, whereas the color map corresponds to the simulation non-GOL) simulations in order to simplify the comparison.

the potential field extrapolated from $z=0 \mathrm{Mm}$. The magnetic free energy is calculated by subtracting the magnetic energy from a potential field extrapolation at $z=0 \mathrm{Mm}$ from the total magnetic energy and averaging this through the horizontal axis. The GOL simulation tends to accumulate slightly more magnetic free energy in the middle-upper chromosphere and TR $(z=[2,5] \mathrm{Mm})$ but less magnetic free energy in the corona. In other words, the ion-neutral effects appear to prevent free energy from reaching the corona, instead accumulating nonpotential field in the chromosphere, where it is dissipated. The dissipation is in part violent and leads to the increased thermal and kinetic energy we find in the GOL simulation (in certain locations with timescales $\left[e / Q_{j_{\text {amb }}}\right]$ of a few seconds or even shorter). There are several reasons for this (see also the following sections):

1. Our simulations do not include imposed flux emergence through the bottom boundary; however, the convective motions cause horizontal magnetic fields to accumulate in the subadiabatic photosphere (see Section 4.5.3). Strong concentrations of magnetic flux can lead to a reduced gas pressure that may facilitate the onset of the RayleighTaylor instability (RT, which is caused by denser plasma overlying less dense plasma). However, the photosphere is subadiabatic and therefore has strong stabilizing properties (Acheson 1979; Archontis et al. 2004). As suggested by Leake \& Arber (2006) and Leake \& Linton (2013), the ion-neutral interaction facilitates the diffusion of magnetic field through the photosphere (Acheson 1979), allowing more magnetic flux to penetrate into the chromosphere than what occurs in the non-GOL simulation.

2. However, ambipolar diffusion does not always facilitate the diffusion of magnetic field into the chromosphere. One way to visualize this is to consider Equation (4), in which the ambipolar term in the induction equation is written as an advection term. The ambipolar velocity diffuses magnetic field into the chromosphere if it is oriented upward and removes chromospheric field when it is directed downward. The latter occurs when the (horizontal) magnetic field strength increases with height, which will lead to downward ambipolar velocities.

3. The ambipolar diffusion can not only diffuse magnetic field but also concentrate magnetic flux, which will help to promote RT instability due to buoyancy. This can be explained as follows: in cases with a horizontal flux tube with twist, in the upper part of the tube, the orientation of the current and magnetic field lines, following the righthand rule, will always lead to an ambipolar velocity pointing toward the convection zone. On the contrary, in the lower part of the tube, the current points in the same direction as in the upper part of the tube while the magnetic field lines are oriented in the opposite direction, so the ambipolar velocity will push the magnetic field lines toward the corona (see Sections 4.4, 4.5.2, and 4.5.3). In other words, ambipolar diffusion tends to compress a horizontal twisted flux tube. This leads to a decrease of the gas pressure and thus an increased buoyancy.

4. We find more magnetic free energy in the middle-upper chromosphere and TR due to the excess magnetic flux that expands from below as a result of ambipolar diffusion.

5. Ambipolar diffusion tends to accumulate current in narrow layers. This leads to faster reconnection rates and larger magnetic tension in the chromosphere.

6. Since the chromosphere transforms magnetic energy into thermal and kinetic energy more efficiently owing to ambipolar diffusion, the corona in the GOL simulations contains less magnetic free energy than the non-GOL simulation. 


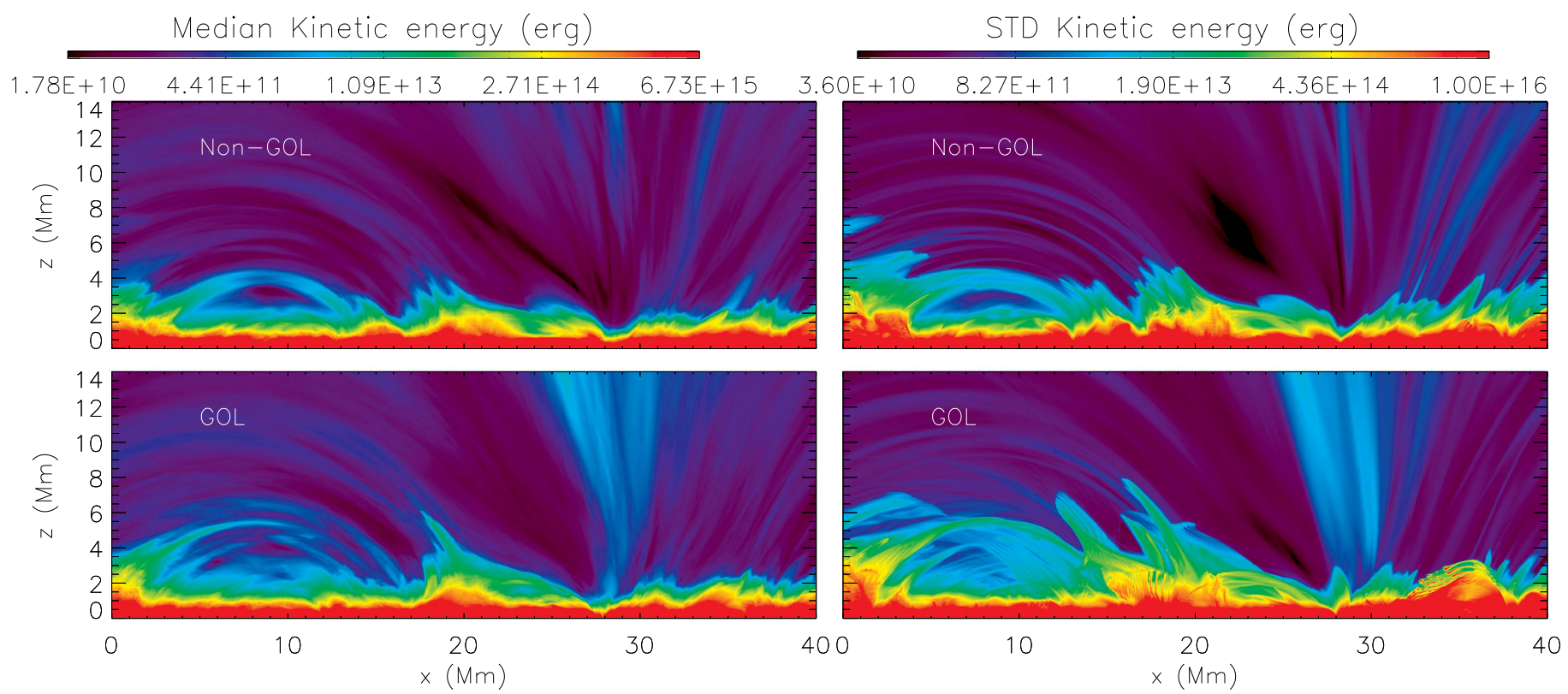

Figure 4. Median (left panels) and standard deviation (right panels) of the kinetic energy (averaged over 11 minutes, snapshots $=[300-370]$ ) for the non-GOL (top panels) and GOL simulations (bottom panels), for $x=[0-40] \mathrm{Mm}$.

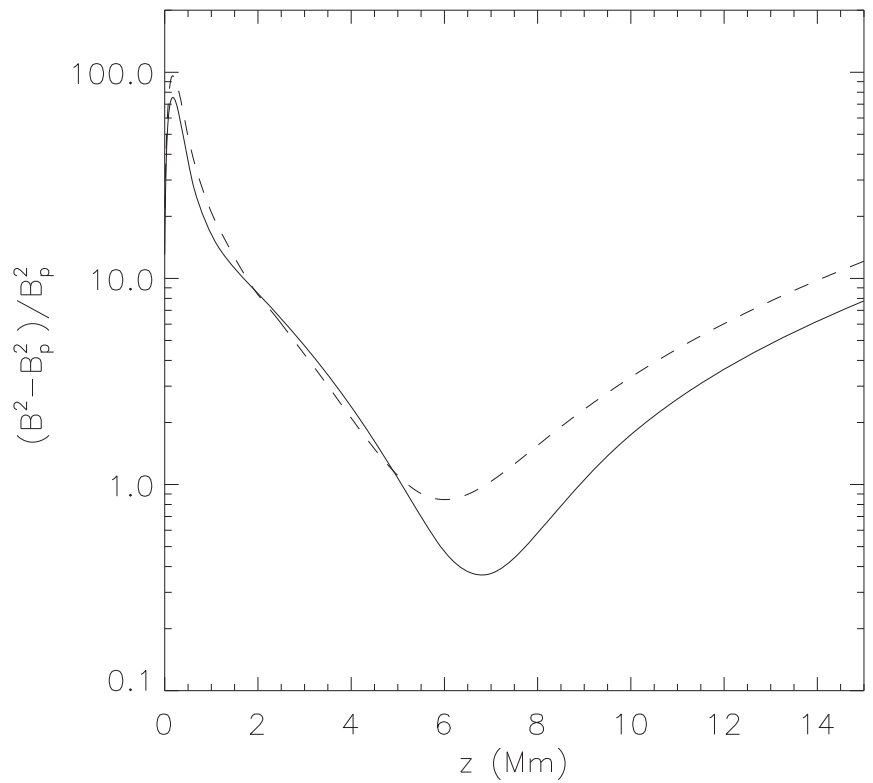

Figure 5. Magnetic free energy normalized by the magnetic energy of the potential field extrapolated from $z=0 \mathrm{Mm}$ for the GOL (dashed) and nonGOL simulations (solid) integrated over 11 minutes (snapshots $=[300-370]$ ).

We also find that as a result of the ambipolar "velocity" thermodynamic structures may decouple from the magnetic field under certain circumstances. When ambipolar diffusion, magnetic field strength, and the current perpendicular to the magnetic field are high enough and the timescales of the thermodynamic processes are longer than the ambipolar processes, the thermodynamic structuring does not necessarily follow the magnetic field direction. One example of this is shown in Figure 6. Before the ambipolar velocity decouples the magnetic field structures from the thermal properties, one can see that the magnetic field collimates the jets and that the TR loops also follow the magnetic field direction (left panels). Later in time, the magnetic field lines rooted at $x=12 \mathrm{Mm}$ move from right to left, and in the lower photosphere the
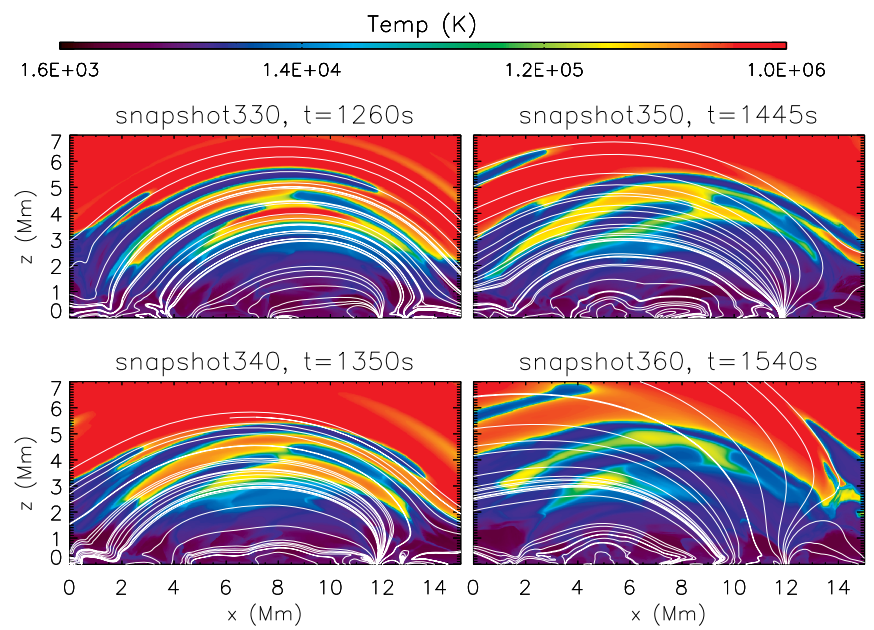

Figure 6. Temperature, in logarithmic scale, for the GOL simulation at $t=1260 \mathrm{~s}$ (top left), $1350 \mathrm{~s}$ (bottom left), $1445 \mathrm{~s}$ (top right), and $1540 \mathrm{~s}$ (bottom right) with magnetic field lines shown in white. In the beginning (left panels) the thermodynamic structures are aligned with the magnetic field, whereas at later times (right panels) the magnetic connectivity has changed and the alignment is poor (see corresponding Movie 1).

(An animation of this figure is available.)

magnetic field connectivity changes so that the magnetic field lines connect to different photospheric locations. The evolution of the magnetic field connectivity is faster than or of the same order as the thermodynamic evolution. As a result, the magnetic field no longer aligns well with the thermodynamic structures in the upper chromosphere and TR. For example, the magnetic field lines cross the jet diagonally on the left side of the bottom right panel. This will impact the evolution of the features (see the drift toward the left of the left magnetic field footpoint in the GOL simulations in the Movie 1). MartínezSykora et al. (2016) describe in more detail the misalignment of the magnetic field lines with thermal structures. These results may provide an explanation for the puzzling observations of de la Cruz Rodríguez \& Socas-Navarro (2011) and Asensio 


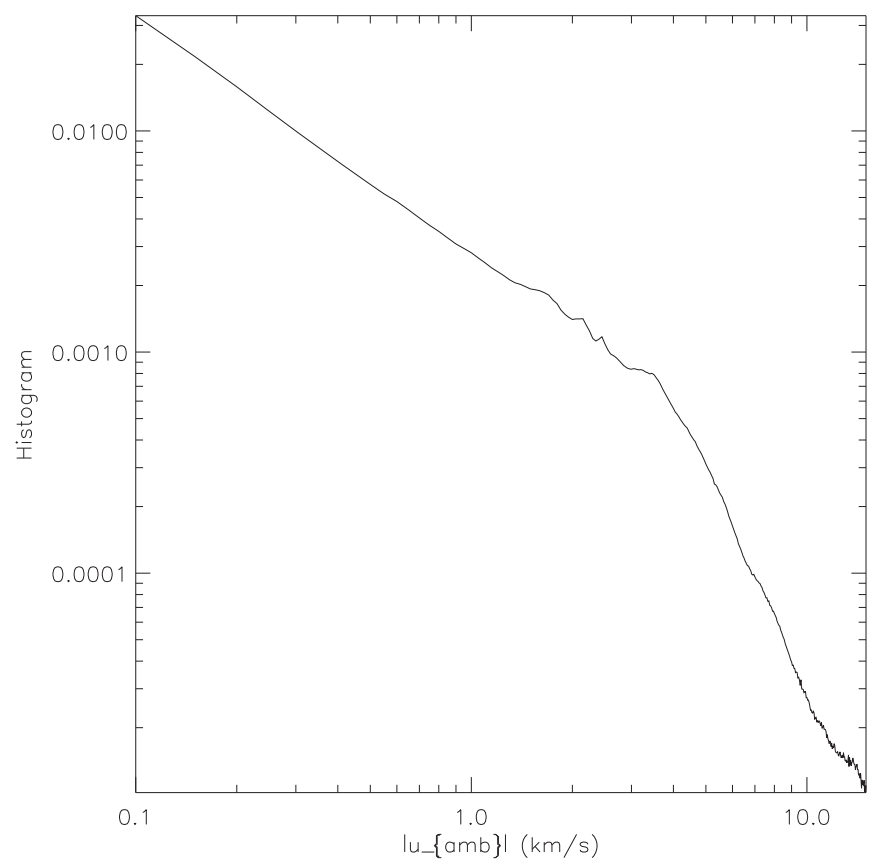

Figure 7. Histogram of the absolute value of the ambipolar velocity $\left(\left|u_{\mathrm{amb}}\right|\right)$, integrated over 11 minutes (snapshots $=[300-370]$ ), revealing a two-step power law (with a slope of -0.07 at low ambipolar velocities and -0.2 at high ambipolar velocities).

Ramos et al. (2017), where fibrils appear to not necessarily follow the magnetic field structures.

The term in the induction equation that leads to the misalignment is that of the ambipolar velocity. For the plagelike magnetic field configuration that we simulate here, a histogram of the ambipolar velocity shows a power-law-like behavior in two distinct regions (see Figure 7). The power-law slope for the lower values of the ambipolar velocity is due to the processes happening in the lower chromosphere, where the plasma is denser and the ambipolar diffusion is smaller. This region is dominated by the shocks driven by the convection zone and the photospheric overshooting. Here the ambipolar velocity distribution is smoother and the locations where the ambipolar velocity is significant are more spatially extended than in the upper chromosphere (where they are more concentrated spatially). In contrast, in the upper chromosphere, the ambipolar velocity is larger and concentrated in narrower regions along the spicules or loops.

\subsection{Heating Properties}

The most obvious difference between these two simulations and previous $2 \mathrm{D}$ simulations is that the convective motions below and up to the photosphere stress the magnetic field sufficiently to self-consistently maintain a hot corona (Figure 1). Previous 2D MHD simulations (Heggland et al. 2011; Leenaarts et al. 2011; Iijima \& Yokoyama 2015; Nóbrega-Siverio et al. 2016) have required a hot plate at the upper boundary in order to produce a hot corona. Previously it was only when computing 3D models that self-consistently heated coronae arose (e.g., Gudiksen \& Nordlund 2002; Hansteen et al. 2010, 2015; Martínez-Sykora et al. 2011; Carlsson et al. 2016). In the current simulations, we find that despite the 2D limitation, the large-scale magnetic field configuration in the current simulation leads to a selfconsistently heated corona. This comes about as a result of the large variety of processes that occur simultaneously within the simulated domain.

In fact, the Joule heating in both of these two simulations not only is greater than in previous 2D models but also extends over a wider range of heights. In previous simulations, most of the heating per particle was strongly confined to the TR. This seems to be in accordance with Hansteen et al. (2015), who suggested that large-scale connectivity leads to a larger scale height for the heating per particle. Our configuration has a smaller decay of Joule heating as a function of height than those smaller-scale simulations in Hansteen et al. (2015).

In order to interpret the various heating mechanisms in these models, it is important to show that regions in which ambipolar diffusion dominates are well resolved:

1. The artificial diffusion and ambipolar diffusion act differently, depending on local magnetic field and thermodynamic conditions (e.g., compare the ambipolar heating in panel (c) with artificial Joule heating in panels (a) and (b) of Figure 8). These two different heating mechanisms are dominant in different regions in the chromosphere (panels (g) and (f) in Figure 8).

2. Our 2.5D simulation reveals that ambipolar diffusion is very important in the chromosphere (panel (f) in Figure 8). In extended regions in the chromosphere, ambipolar diffusion is much larger than the artificial diffusion (compare panels (f) and (f) in Figure 8).

3. The GOL and non-GOL models differ in their magnetothermodynamic properties as detailed in previous sections (Figure 3).

The ambipolar diffusion strongly depends on the thermal properties of the chromosphere and TR. This behavior cannot be captured with $1 \mathrm{D}$ semiempirical models because those do not capture the dynamics of the chromosphere, e.g., shocks, or the significant horizontal spatial structuring. The large variations in ambipolar diffusion have significant consequences for a variety of physical processes (Sections 4.5.1-4.5.3). This large variability of the ambipolar diffusion is due to the significant changes in the ion-neutral collision frequency, which depends on the neutral density, the ionization state, and the temperature.

In terms of absolute values, the ambipolar diffusion term is the largest when comparing to the ohmic diffusion, Hall term, and artificial diffusion. This is shown in panels (d)-(g) in Figure 8. The ohmic diffusion (panel (d)) is larger in the photosphere and chromosphere than in the corona. The Hall term (panel (e)) is important in the corona and in the coolest areas in the chromosphere (see Martínez-Sykora et al. 2012, for details). Despite this, the Hall term in our 2.5D numerical model did not reveal any appreciable impact on the simulated atmosphere. Most likely one may need to expand the simulation to three dimensions, or include greater spatial resolution (e.g., to resolve Whistler waves; e.g., Huba 2003).

The Joule heating caused by ambipolar diffusion in the GOL simulation (panel (c) of Figure 8) is mostly localized in two types of regions: (1) The cold chromospheric bubbles produced by the rarefaction in the wake of shocks that pass through the chromosphere. This heating is larger in the upper regions of the cold expanding bubbles, where the density, temperatures, and ion-neutral collision frequency are lower. The ambipolar heating is not very important in denser regions, i.e., close to the photosphere. (2) The ambipolar diffusion is also very important in the upper chromosphere. There we find large 

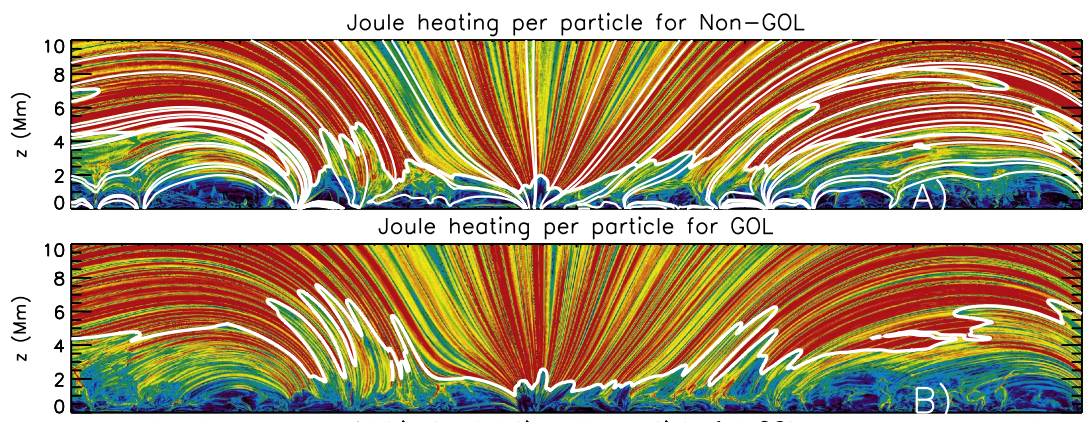

Ambipolar heating per particle for $\mathrm{GOL}$
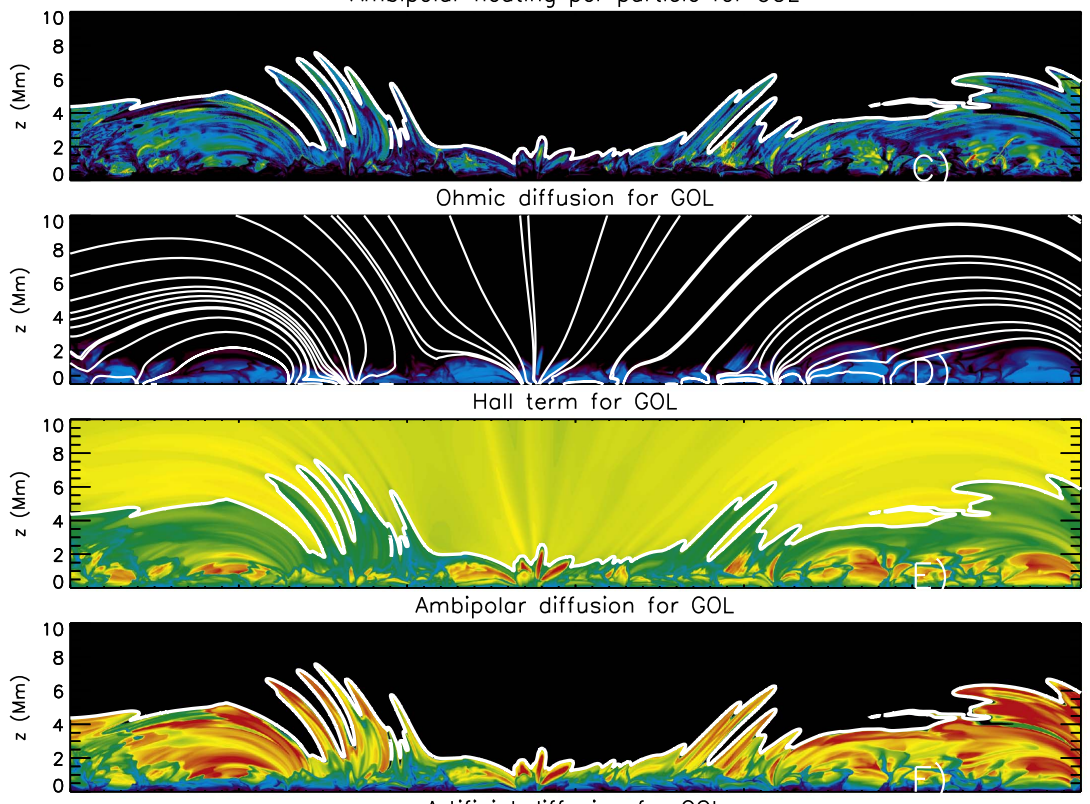

Artificial diffusion for GOL
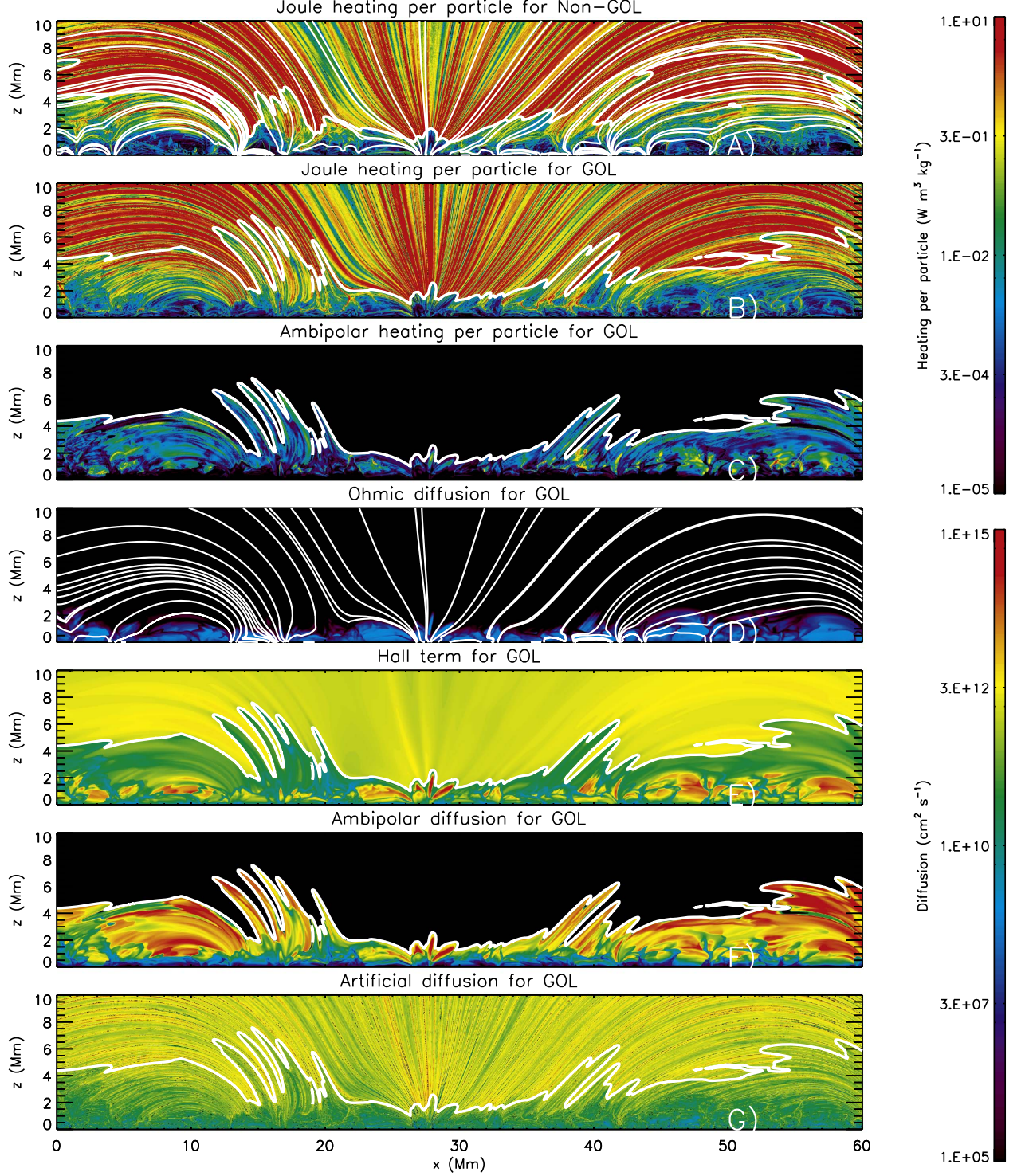

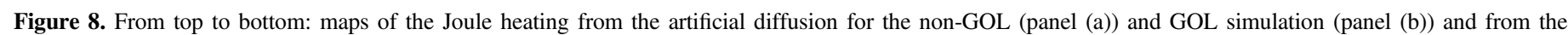

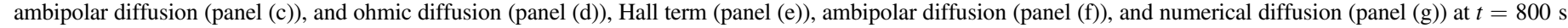

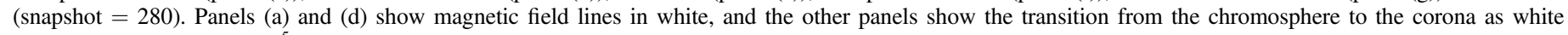

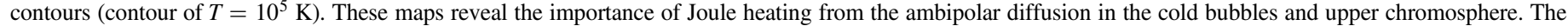
color schemes are in logarithmic scale.

spicule-like extrusions of the TR that roughly align with the inclined magnetic field (regions between $x=$ [13-23] Mm and $x=[37-43] \mathrm{Mm})$. These are heated by ambipolar diffusion. Similarly, the upper chromosphere in regions with highly inclined (almost horizontal) magnetic field are also heated by ambipolar heating (regions between $x=[0-10] \mathrm{Mm}$ and $x=[45-60] \mathrm{Mm})$.

It is interesting to see that in the cold bubbles in the lower atmosphere, where the ratio of neutral to ionized particles is very large (see also Martínez-Sykora et al. 2012), the Joule heating from the artificial diffusion (panel (b)) is smaller than both the same heating term in the non-GOL simulation (panel (a)) and the Joule heating from the ambipolar diffusion (panel (c)). However, in the upper chromosphere, TR, and lower corona the Joule heating from the artificial diffusion (panel (b)) is larger than in the non-GOL simulation (panel (a)). This is caused by a combination of two effects: (1) the ambipolar diffusion allows more magnetic flux to reach into the chromosphere, which, through interaction with the ambient, preexisting field, leads to more heating (see Sections 4.4 and 4.5.3); and (2) the ambipolar diffusion concentrates the electrical current, which is dissipated by both ambipolar diffusion and artificial diffusion.

\subsection{Magnetic Flux Transport}

Sporadically the ambipolar diffusion releases photospheric magnetic field into the chromosphere. Since the Hall and ambipolar terms can be rewritten as in Equation (4), they may be interpreted as a Poynting flux where the velocities are the Hall and ambipolar "velocities," respectively. Therefore, one can calculate the vertical Poynting flux owing to vertical and horizontal components of the ambipolar velocity:

$$
\begin{gathered}
\boldsymbol{P}_{z z} \equiv \boldsymbol{u}_{A_{z}}\left(B_{x}^{2}+B_{y}^{2}\right), \\
\boldsymbol{P}_{z h} \equiv \boldsymbol{B}_{z}\left(B_{x} u_{A x}+B_{y} u_{A y}\right),
\end{gathered}
$$




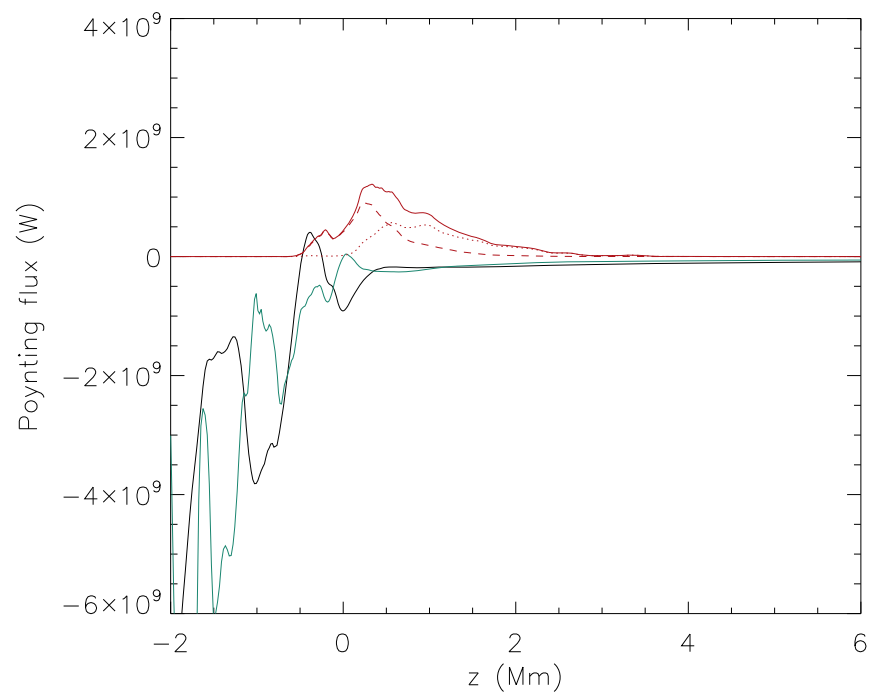

Figure 9. Total vertical Poynting flux of the advection term for the non-GOL (black) and GOL simulations (green) as a function of height. All values are averaged in time (over 1.5 minutes, snapshots $=[360-370]$ ) and in the horizontal direction. The vertical Poynting flux due to the ambipolar diffusion in the GOL simulation (red) is shown as a function of height. The dashed line is for horizontal motions $\left(P_{z h}\right)$, the dotted line is for vertical motions $\left(P_{z z}\right)$ and the solid line is the sum of both.

which are shown in Figure 9 as red dotted and red dashed lines, respectively. The sum of both is shown with the solid red line. This figure also shows the vertical Poynting flux (only the contribution from the advection) for the non-GOL (black) and GOL simulations (green) as a function of height. The values are integrated over a time period of 2 minutes.

Ambipolar diffusion can facilitate the transport of magnetic field from the photosphere into the chromosphere by $\sim 10^{9} \mathrm{~W}$. This does not occur universally since the effect is concentrated in a few locations or events instead of being uniformly distributed over the upper photosphere. (See the example in Section 4.5.2.) The Poynting flux due to the ambipolar "velocity" is important in the proximity of the photosphere as can be appreciated from the integrated $\boldsymbol{P}_{z z}$ and $\boldsymbol{P}_{z h}$ as a function of height in dashed and dotted red lines. As a result, in some colder-than-average locations additional magnetic flux is carried into the middle chromosphere. At these locations the magnetic field expands quite rapidly, and this expanding magnetic field interacts with the ambient magnetic field (see Sections 4.5.2 and 4.5.3). This is similar to what Leake \& Arber (2006) found in their 2D simulations of flux emergence, where partial ionization effects were seen to facilitate the expansion of the magnetic field into the upper solar atmosphere and corona. However, there are two major differences between our and their simulations: our simulations do not include any imposed flux emergence, and the ambipolar diffusion is critical for the horizontal field to penetrate into the chromosphere. In contrast, in Leake \& Arber (2006) emerging flux crosses the photosphere owing to the strong buoyancy, while ambipolar diffusion did not play a large role. In their case ambipolar diffusion was not strong in the upper photosphere, as they used a 1D semiempirical model (which does not capture the extremely low temperatures in the wake of shocks) to describe the upper photosphere. When the temperature and ionization dependence of the ambipolar diffusion are taken into account, as in our model, large variations of the ambipolar diffusion (Figure 8) occur at photospheric heights.
The rapid expansion due to the emergence of flux through the photosphere produces cool voids. This occurs in denser regions (blue regions in the GOL simulation for $-10<$ $\log \rho<-7$, Figure 3) than the cold bubbles that arise as a result of rarefaction behind magnetoacoustic shocks $(\log \rho<-10)$. These types of voids are quite rare for the following reasons: (1) the ambipolar diffusion is rarely significant enough in the vicinity of the photosphere to trigger the instability that leads to flux emergence, and (2) we do not include any explicit flux emergence. As a result of the increased flux emergence from the ambipolar diffusion, the magnetic field strength in the photosphere is slightly reduced compared to the non-GOL simulation.

\subsection{Detailed Description of the Various Physical Processes}

We will now describe some of the dominant physical processes in these simulations and how they differ between the two simulations. Our large-scale models reveal, for the first time in this type of model, that the magnetic field configuration and connectivity play a key role in the nature of the dominant magnetothermodynamic processes in the various regions of the domain. We can distinguish several different types of regions in Figure 2: (1) open field lines in the two (opposite-polarity) plage regions (at $x=[25-35] \mathrm{Mm}$ and at $x \sim[60-80] \mathrm{Mm}$ ), dominated by magnetoacoustic shocks (dynamic fibrils); (2) inclined magnetic field lines that penetrate into the corona and connect both plage regions next to the open field lines $(x=[19-25] \mathrm{Mm}, \quad x=[35-50] \mathrm{Mm}, \quad x=[67-70]$, and $x=[80-96] \mathrm{Mm}$ ), dominated by taller spicules and jets (note the overlap between various regions due to the complexity of the connectivity); (3) regions where the chromospheric magnetic field lines are highly inclined or almost horizontal and never reach the corona $(x=[2-18] \mathrm{Mm}$ and $x=[50-60]$ $\mathrm{Mm})$. The latter are located in regions between the two polarities. The physical processes that we will describe in detail are the magnetoacoustic shocks, chromospheric jets, and the reconnection processes that occur in regions with highly inclined magnetic field. The different spatiotemporal evolution of these processes between the two simulations is ultimately the cause for the different thermodynamic stratifications that we described in the previous sections.

\subsubsection{Expanding "Cold” Rarefraction Bubbles}

As a result of the large ambipolar diffusion in the cold bubbles in the wake of strong acoustic shocks, we find differences in several aspects compared to the non-GOL simulation. This is true not only for the thermal properties but also for the magnetodynamic properties of these bubbles. Ambipolar diffusion reduces, and in many cases removes, the current density in the inner upper part of the bubble through dissipation into thermal energy. The cold bubbles in the nonGOL simulation typically show significant current throughout (panel (j), Figure 10). This is not the case in the GOL simulation, where the current density is typically removed owing to ambipolar diffusion, especially in the upper part of the cold bubbles (panel $(\mathrm{k})$ ). As a result, the magnetic field in the GOL simulation tends to be nearer to potential and more uniformly distributed in the cold chromospheric bubbles, despite their strong expansion. Since the magnetic field is less uniformly distributed in the non-GOL simulation, we find the lowest magnetic field strengths in the cold bubbles in the non- 

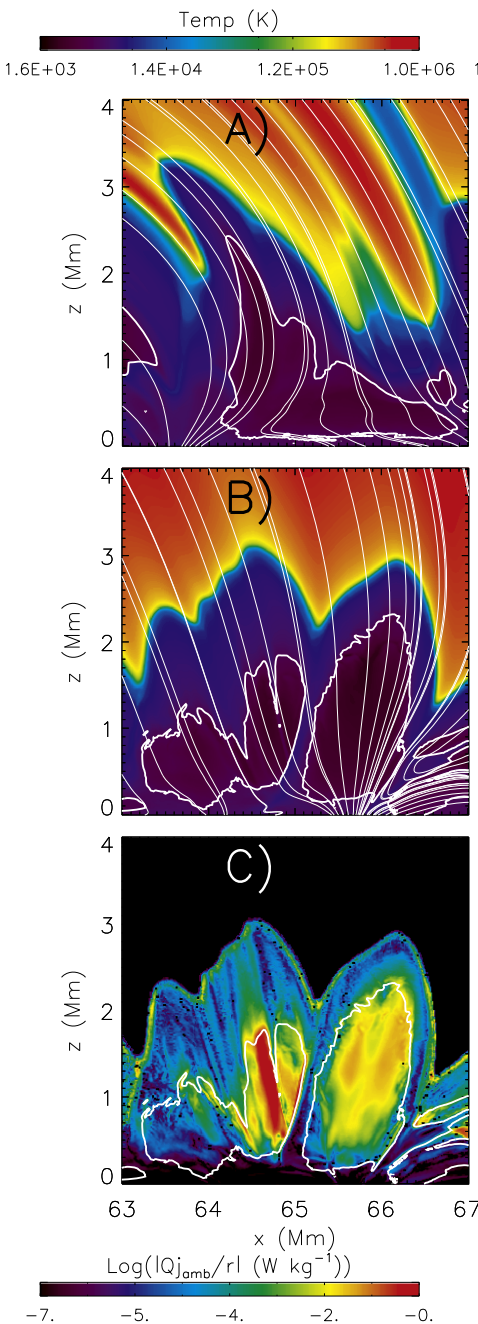
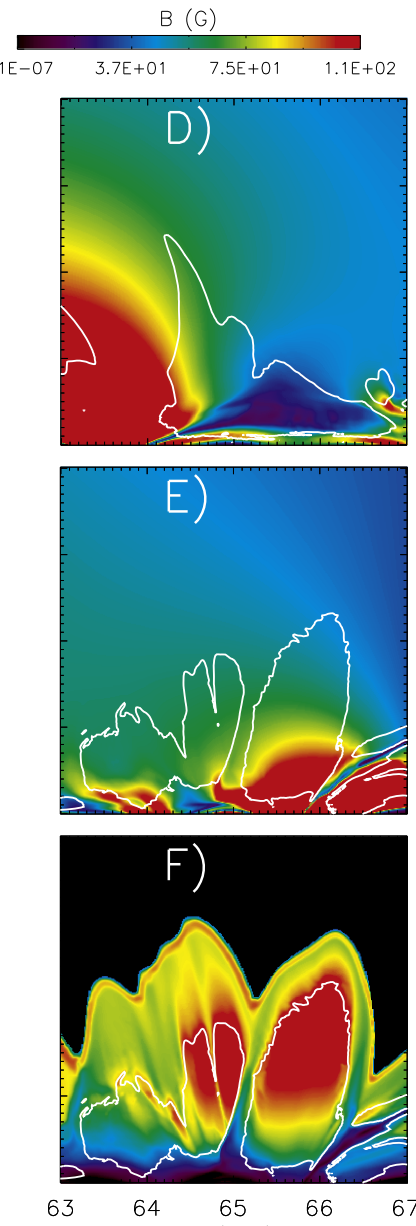

$\times(\mathrm{Mm})$

$\log \left(\left|\eta_{\mathrm{omb}}\right|\left(\mathrm{cm}^{2} / \mathrm{s}\right)\right)$
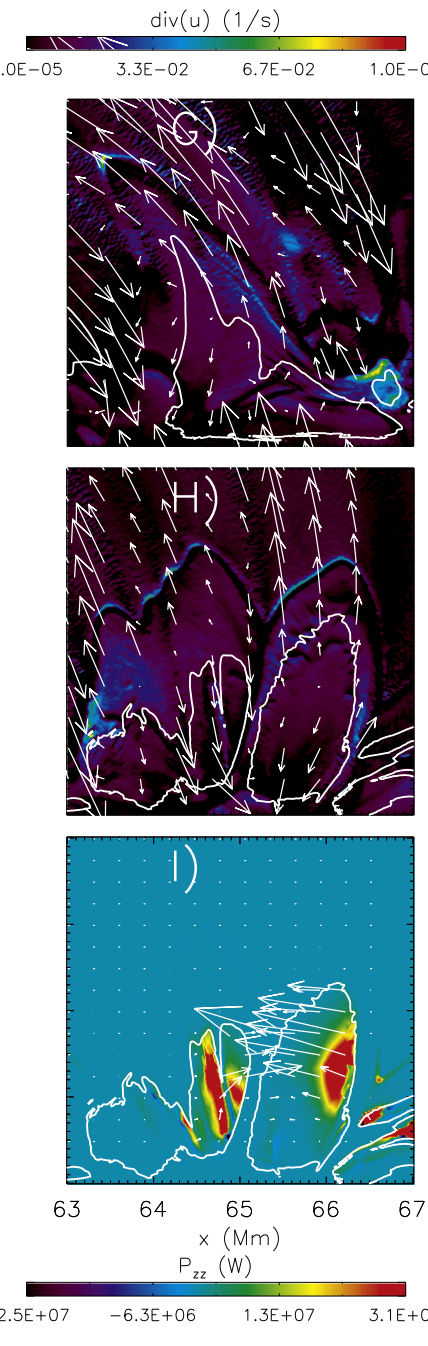

$-6.3 \mathrm{E}+06$
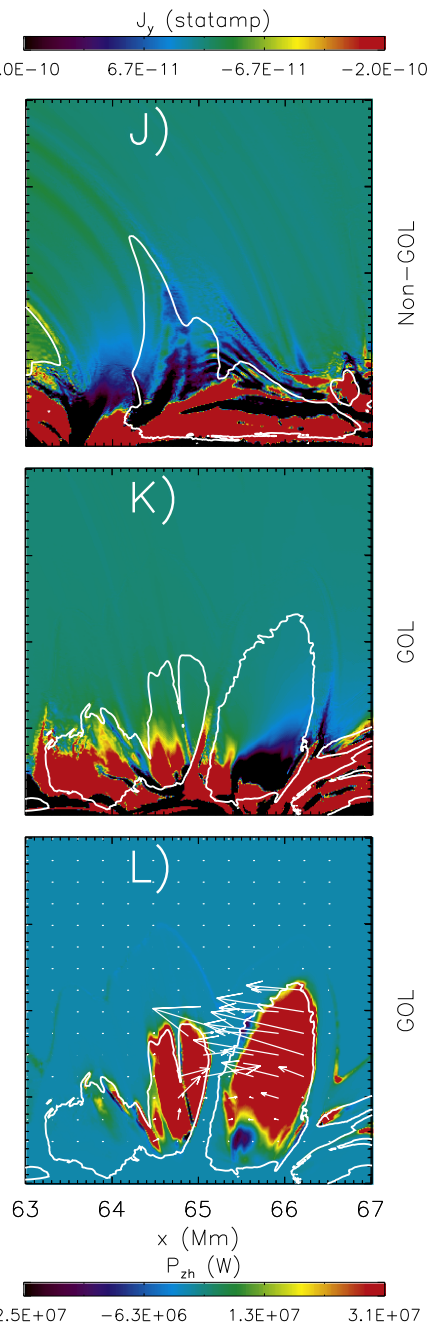

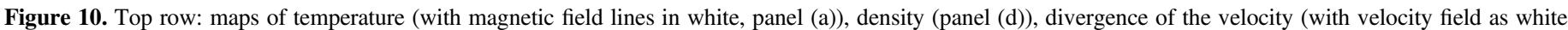

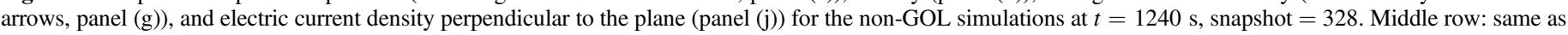

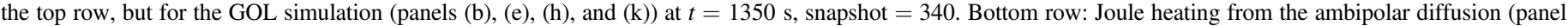

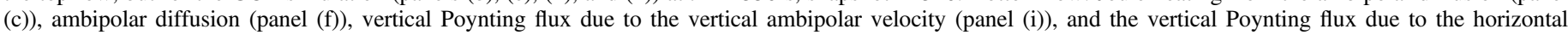

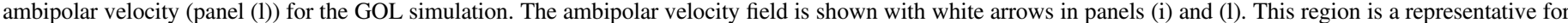

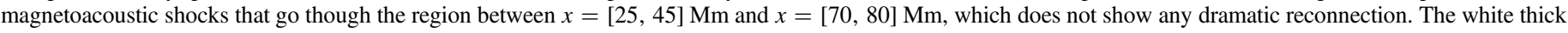
contours correspond to a $4000 \mathrm{~K}$ temperature.

GOL simulation (e.g., compare the bubbles at $x \sim 65 \mathrm{Mm}$ in panel (d) of Figure 10 with the ones at $x=65-66 \mathrm{Mm}$ in panel (e)). In the non-GOL simulation we also see that in regions with predominantly vertical fields the magnetic field strength shows stronger variations than in the GOL simulation (not shown in the figure).

Note that not all cold bubbles are characterized by significant ambipolar diffusion, as this depends on the bubbles' density, ion-neutral collision frequency, and magnetic field strength. If the ambipolar diffusion is strong, we find that at later stages of a bubble's evolution the current density is entirely removed from the upper part of the bubble and the remaining current tends to be located at the sides of the bubble (see at $x=66.5$ $\mathrm{Mm}$ in panel (k)). The current density is concentrated there as a result of the interaction between the magnetic field in the expanding bubble, which suffers from the strong ambipolar diffusion, and its surroundings (see the ambipolar diffusion in panel (f) of Figure 10).

The expanding cold bubbles found in the GOL simulation are warmer (Figure 3) as a result of the increased magnetic field dissipation caused by ambipolar diffusion (see panels (c) and (f) in Figure 10).

It is also illustrative to consider the ambipolar velocity field, which is shown in panels (i) and (l) with white vectors for the GOL simulation.

In the spicules, which are mostly vertical, the ambipolar velocity is almost horizontal because the magnetic field is vertical and the current is perpendicular to the plane of the simulation. Therefore, the ambipolar advection of the magnetic field lines leads to the leftward drift of the field lines of the leftmost plage region in the GOL simulation, which explains the horizontal drift of the magnetic field shown in Movie 1, Figure 6 and Section 4.2.

\subsubsection{Chromospheric Jets}

We find several jets that arise from expansion of magnetic field lines into the chromosphere. These jets are considerably larger and faster in the GOL simulation than in the non-GOL simulation. The jets in the GOL simulation share many similarities with the so-called type II spicules. Their formation 

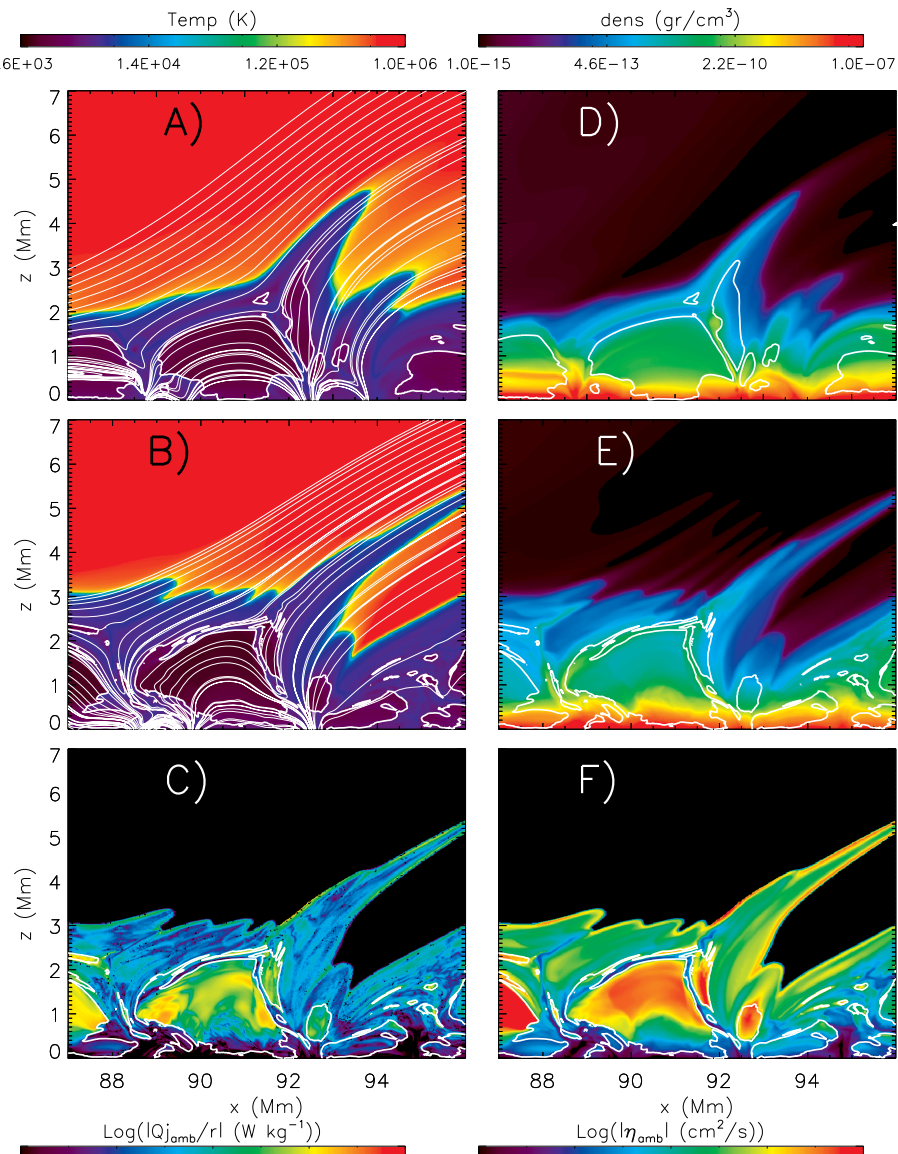
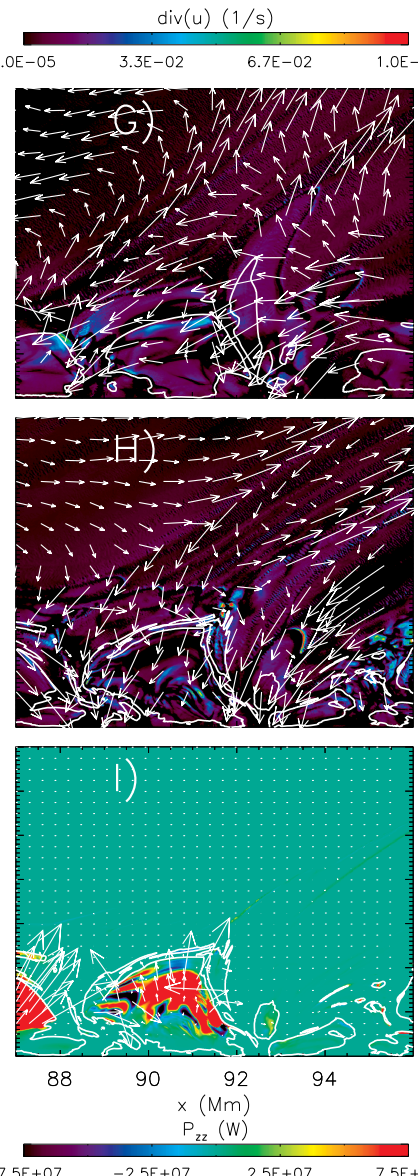
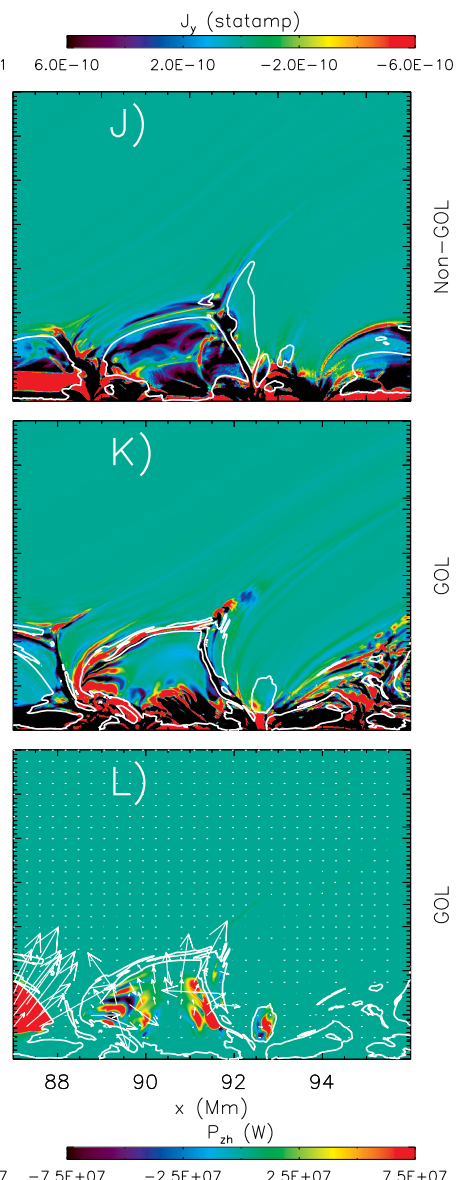

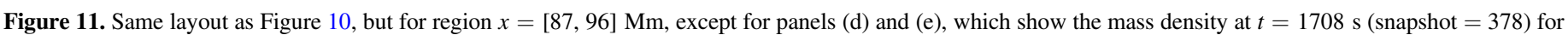
the non-GOL simulation and $t=1360 \mathrm{~s}$ (snapshot $=341$ ) for the GOL simulation.

mechanisms and comparison with observations are investigated in more detail in a separate paper (Martiunez-Sykora et al. 2017a). In addition to the similarities in their length $(\sim 8 \mathrm{Mm})$ and speed $\left(\sim 100 \mathrm{~km} \mathrm{~s}^{-1}\right)$, the magnetic field strength along the spicule also seems to be in accordance with observations (compare Figure 1 with Orozco Suárez et al. 2015, Figure 4).

In short, the formation of many of these jets is caused by the expansion of magnetic field into the chromosphere. When the ambipolar diffusion is large enough in the lower chromosphere and upper photosphere, the magnetic field can diffuse through the subadiabatic photosphere (Leake \& Arber 2006). We find that this occurs frequently in the vicinity of strong flux concentrations when they interact with granular scale fields in their vicinity. Once through the photosphere, the magnetic field will expand, producing a cold void characterized by low temperatures in the lower chromosphere (Figure 3). ${ }^{6}$ In contrast, these weak-field regions do not pass into the chromosphere as often in the non-GOL simulation since it lacks ambipolar diffusion.

The cold voids are a crucial step in the formation of the jets. As we describe below, these voids are completely different

\footnotetext{
6 In fact, it is only in these regions in the GOL simulation that the ad hoc heating term is playing some role. In contrast, the ad hoc heating plays a key role in many more regions in the non-GOL simulation. To take this into account in the GOL simulation, we changed the threshold temperature for the ad hoc heating term from 1600 to $1800 \mathrm{~K}$ at $t=1460 \mathrm{~s}$ ( snapshot $=352$ ). We note that the ad hoc heating is below the one needed for the non-GOL simulation $(2000 \mathrm{~K})$. This should be taken into account while analyzing the simulation snapshots that are being provided.
}

from the cold bubbles that occur in the wake of propagating shocks (described in Section 4.5.1). Once again, ambipolar diffusion plays a critical role in the evolution of the cold voids. The GOL simulation shows that the ambipolar diffusion is very high in these cold regions, so that the diffusion occurs on short timescales $\left(\Delta t_{A}\right)$. This means that the magnetic field in these cold voids diffuses on timescales shorter than the lifetime of the voids. In the non-GOL simulation this diffusion does not occur, and the nearly frozen-in magnetic field is significantly reduced as the plasma expands. One example is shown in Figure 11: the expanding cold void in the GOL simulation $([x, z] \sim[91.5,1]$ $\mathrm{Mm})$ shows fairly strong currents and fairly strong magnetic field. Magnetic field diffuses in the cold void, and the current is concentrated at the exterior boundaries of the void (panel (k) in Figure 11).

Upon expansion in the chromosphere, the magnetic field interacts with the ambient field, which is inclined and penetrates into the corona. It is the release of the confined magnetic tension (confined because of the presence of ambipolar diffusion) that drives the strong flows that lead to spicular jets. As a result of these thin current layers, the magnetic tension is much larger than in the non-GOL simulation. Therefore, more magnetic energy is released into kinetic and thermal energy (compare panels (j) and (d) in Figure 11 and also the examples shown in Figure 12). Thus, with a larger magnetic tension, spicule outflows are longer and faster in the GOL simulation (see the jets that occur at the edges of the two plage polarities in Figure 8). 


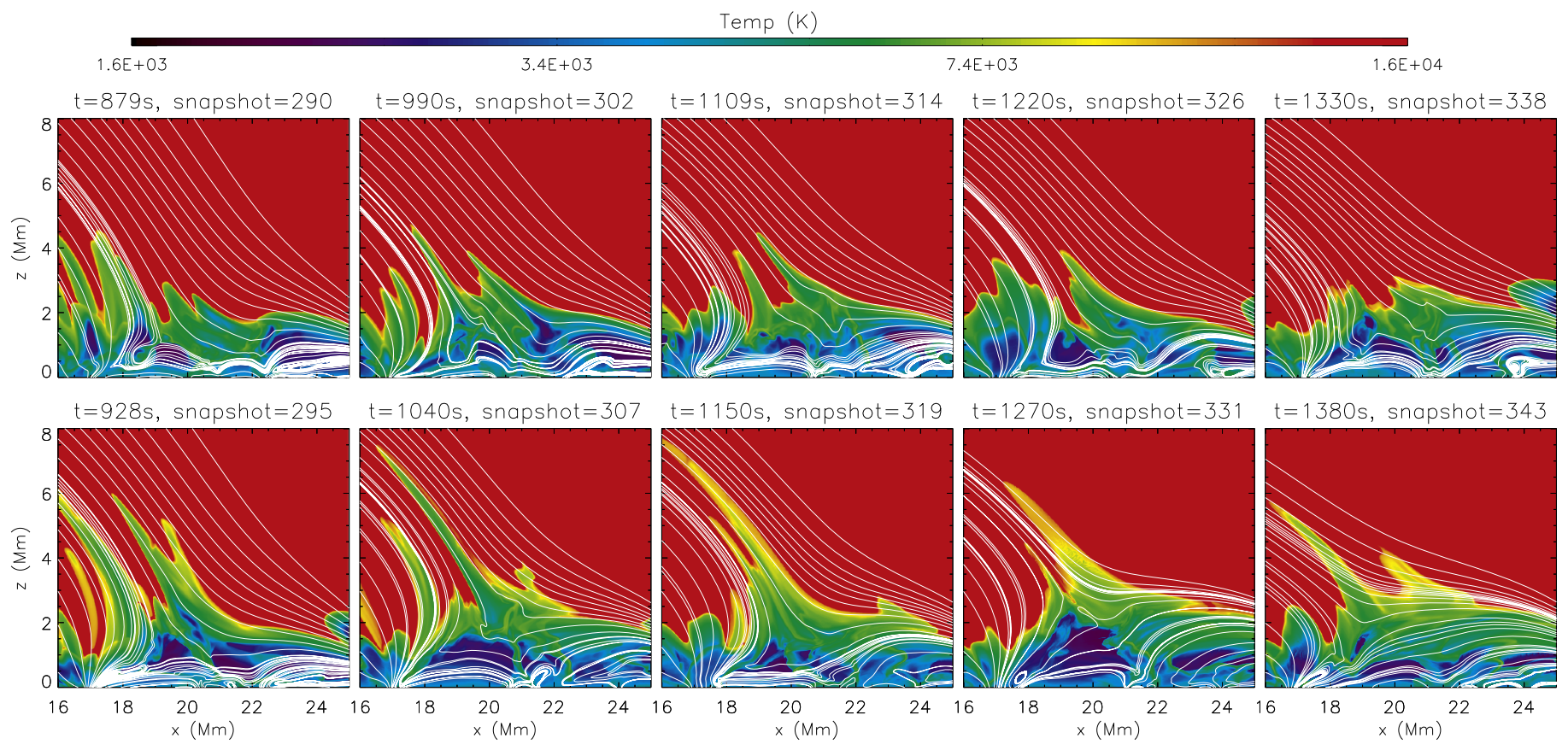

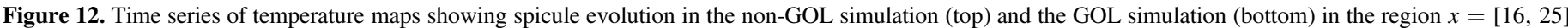
$\mathrm{Mm}$. Magnetic field is drawn with white lines (see the corresponding Movie 2).

(An animation of this figure is available.)

Figure 12 shows temperature maps for the non-GOL (top) and GOL simulations (bottom). There are three major differences between the simulations: (1) the spicules are longer and faster in the GOL simulation; (2) magnetic field lines seem to decouple from the thermodynamic structure of the spicule near the end of their evolution in the GOL simulation (bottom right panel); and finally, (3) the spicules are heated by ambipolar dissipation in the GOL simulation and the temperature therefore increases with time. This temperature increase does not occur in the non-GOL simulation.

The heating of the spicules in the GOL simulation is of significant interest since it matches well with observations of type II spicules that seem to "disappear" in the Ca II passband but not in Mg II or TR lines (Pereira et al. 2014). Detailed studies show that there is a clear evolution of spicule strands being heated from chromospheric to TR temperatures (Skogsrud et al. 2015). The temperature evolution of the spicules in the GOL simulation agrees well with those observations (Martiunez-Sykora et al. 2017a).

The physical mechanism for heating the spicules includes several components. Figure 11 (from the GOL simulation) illustrates one component: the spicule shown carries current perpendicular to the plane of the simulation that is spread along the spicule axis. This current is dissipated through ambipolar diffusion, heating the spicule to TR temperatures despite the strong adiabatic expansion (see Movie 2 and the time series in Figure 12). The rapid expansion actually plays a key role in heating the spicular plasma: it leads to a rapid drop in density so that the ambipolar diffusion becomes larger (especially toward the spicule tops) since the ion-neutral collision frequency is smaller. Since the spicule density is low compared to deeper regions in the chromosphere, ambipolar heating is so effective that it can more than compensate for the adiabatic cooling from the spicule expansion. There is also a second heating component: there is additional heating through ambipolar dissipation of the transverse waves that are driven by the release of the magnetic tension. These two heating mechanisms explain the larger density of points in the upper chromosphere and TR of the 2D histogram of the GOL simulation in Figure 3.

The simulated type II spicules also impact the corona in a variety of ways. Shocks pass through, currents penetrate and heat the corona, flows fill the loops, and thermal conduction spreads the resulting thermal energy through the million-degree plasma in the associated loops. As a consequence of this, we see spicules impact various coronal observables. The impact of the type II spicules on the corona and the comparison with observations are described in detail in De Pontieu et al. (2017) and J. Martinez-Sykora et al. (2017b, in preparation).

\subsubsection{Chromospheric Reconnection in Highly Inclined Fields}

Cold bubbles also form in the wake of shocks that propagate in regions that include highly inclined (almost horizontal) magnetic field lines. In some cases, this photospheric horizontal magnetic field may diffuse, i.e., be advected with the help of the ambipolar velocity, into the middle chromosphere (panels (j), (k), (i), and (l) in Figure 13). This is similar to what was described in Section 4.5.2. Thus, the magnetic field expands into the outer atmosphere, which pushes chromospheric material to higher layers. With this magnetic field topology, the magnetic field frequently forms dips in the proximity of intergranular lanes owing to the downflows. As a result of these dips, the field often reconnects with ambient field or magnetic field associated with neighboring cold bubbles (see panels (a), (b), (m) and (n)). Ambipolar diffusion enhances the reconnection rate as it concentrates the current into thinner layers (panels (f), (i), (l), (m), and (n)). Since the magnetic field is highly inclined, this process does not create a jet into the corona, but rather helps to fill the upper chromosphere with plasma (panels (d) and (e)). As a result of these processes, magnetic energy is 

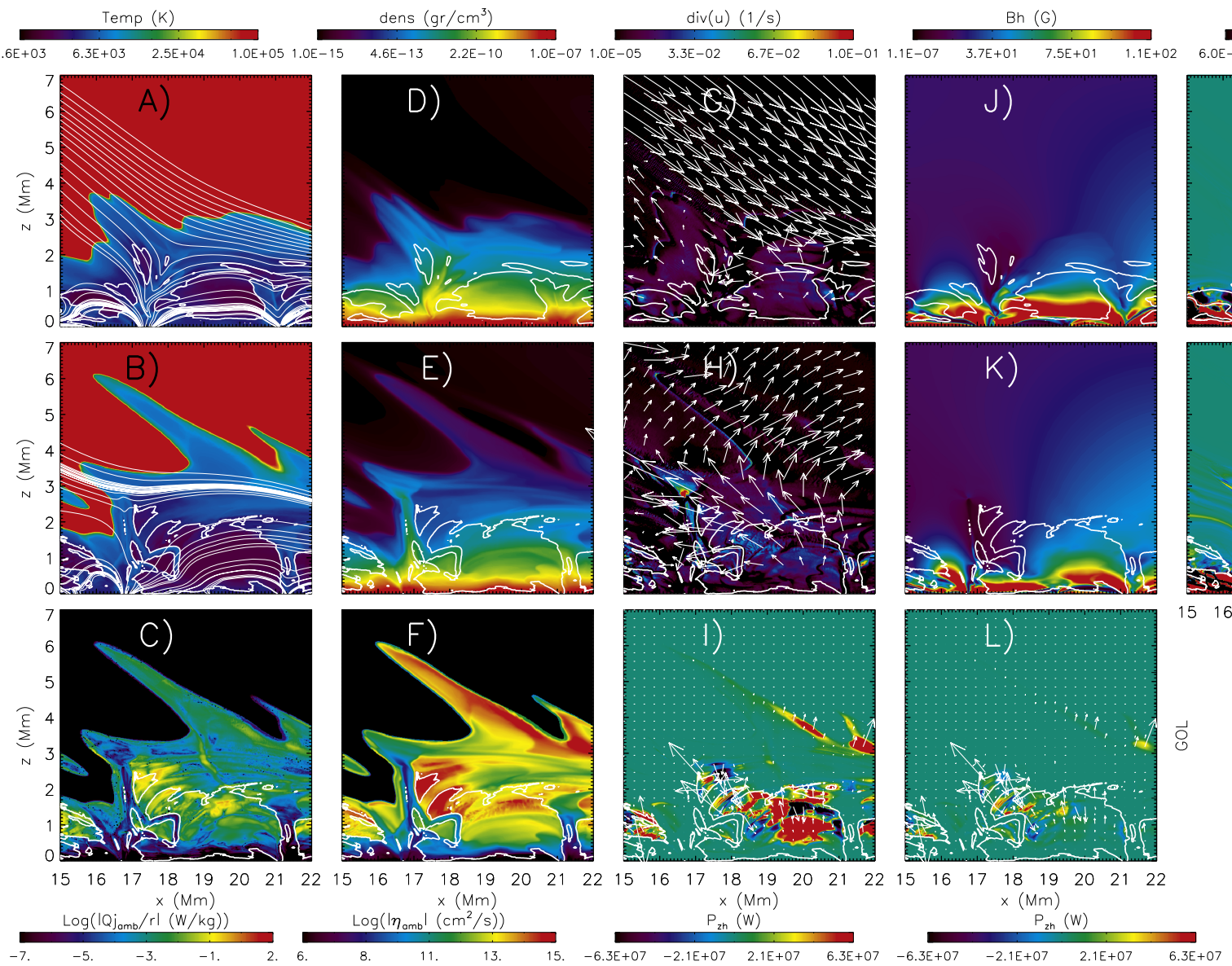

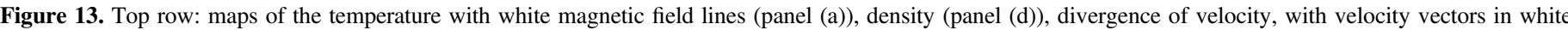

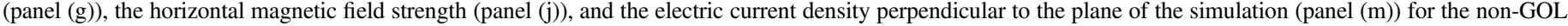

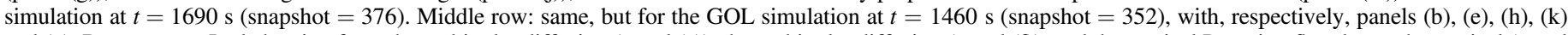

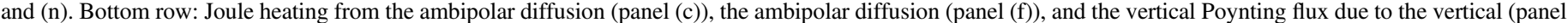

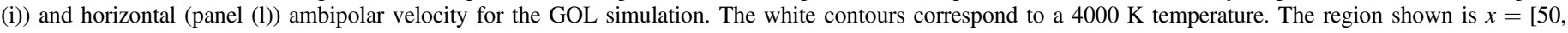
57] $\mathrm{Mm}$ and $x=[15,22] \mathrm{Mm}$ for the non-GOL and GOL simulations, respectively.

dissipated and transformed into thermal energy in the upper chromosphere up to $5 \times 10^{4} \mathrm{~K}$. We also see more kinetic energy in the GOL simulation in the upper chromosphere, TR, and lower corona as a result of the interaction of the diffused magnetic field with the ambient magnetic field. This leads to a wider range of densities in the upper chromosphere, TR, and corona (Figure 3).

We note that the magnetic field topology, where dips in the magnetic field occur, shows some resemblance to the topology thought to play a role in Ellerman bombs (Georgoulis et al. 2002), except that in our case the magnetic field strength is much weaker and the overlying fields are highly horizontal.

\section{Discussion and Conclusions}

We have performed 2.5D radiative MHD simulations of the solar atmosphere using the Bifrost code including ion-neutral interaction effects. These effects are implemented in the code by adding the Hall term and the ambipolar diffusion (Pedersen dissipation) in the induction equation, including a new hyperdiffusive scheme to remove instabilities that may come from these two terms.

Martínez-Sykora et al. (2012) described how the thermomagnetic properties of the chromosphere lead to variations of the Hall term and ambipolar diffusion. In this paper we have detailed the impact of the partial ionization on the thermodynamics and magnetic field evolution.

Before we summarize the differences between the simulations, we focus on a similarity between the two simulations that has not been reproduced in previous 2D self-consistent radiative MHD simulations. Despite the fact that the simulations are $2 \mathrm{D}$, the corona is self-consistently maintained well above a million degrees. Previous 2D simulations required a hot plate at the top boundary in order to maintain a milliondegree corona (e.g., Heggland et al. 2011; Leenaarts et al. 2011; Iijima \& Yokoyama 2015; Nóbrega-Siverio et al. 2016). It was necessary to expand into three dimensions and have closed field lines, i.e., loops, in order to obtain a selfmaintained hot corona (e.g., Gudiksen \& Nordlund 2002, 2005; Hansteen et al. 2010; Martínez-Sykora et al. 2011; Carlsson et al. 2016). Our two 2.5D simulations are different from previous 2D simulations: they are higher resolution and include a larger numerical domain and large-scale magnetic field connectivity. This combination of a large-scale magnetic field and the large variety of physical processes and features that occur throughout the simulated domain is key to produce a hot corona. Some of the key processes that occur in our domain are (1) magnetoacoustic shocks, i.e., type I spicules with a large variety of magnetic field inclinations; (2) fast jets that appear similar to type II spicules; and (3) magnetic reconnection 
within the chromosphere, where the magnetic field lines are highly inclined. All of these processes combined build enough magnetic energy in the TR and low corona and are able to produce enough thermal energy to self-consistently maintain the corona at temperatures well above a million degrees. We also note that the large-scale magnetic connectivity plays an important role in increasing the scale height of the heating per particle compared to previous simulations: the heating now reaches higher into the corona. This seems to be in accordance with the results of Hansteen et al. (2015).

In addition to this similarity between the GOL (which includes partial ionization effects) and non-GOL simulations, there are many differences, from the chromosphere all the way into the corona. In the GOL simulation:

1. The expanding cold chromospheric bubbles are hotter owing to the Joule heating from the ambipolar diffusion when the bubbles reach low enough densities.

2. The upper chromosphere and TR spread over a broader range of heights and densities. Similarly, the corona has a wider range of densities.

3. The upper chromosphere is hotter since the ambipolar diffusion dissipates magnetic energy into thermal energy. This also leads to spicules being heated during their evolution.

4. The kinetic energy in the upper chromosphere, TR, and lower corona is larger and shows a wider range of values as a result of more violent processes.

5. The magnetic free energy in the upper chromosphere and TR is larger than that in the non-GOL simulation by a factor of $\sim 1.3$ for the following reasons: (1) Some horizontal magnetic field that is normally constrained to the subadiabatic photosphere is diffused into the chromosphere. Consequently, the magnetic free energy in the photosphere is reduced compared to the non-GOL simulation. (2) The increased emergence into the chromosphere, combined with the interaction with the ambient magnetic field, leads to more magnetic free energy in the chromosphere. (3) In addition, the boundaries between regions with high and low ambipolar diffusion often have strong variations of field line connectivity.

6. In the corona, in contrast, the magnetic free energy is smaller since the simulated chromosphere is able to convert more magnetic energy into kinetic and thermal energy owing to the ambipolar diffusion.

Flux emergence plays a key role in the GOL simulation, even though we did not impose explicit flux emergence at the boundaries of the numerical domain. Ambipolar diffusion plays a key role in facilitating the emergence of relatively weak magnetic flux that otherwise would not be buoyant enough to expand into the atmosphere. We also found a few locations where the Lorentz force points toward the convection zone, so that the ambipolar diffusion, where it is large enough, helps to move the flux into deeper layers. We found that once the flux has emerged into the chromosphere, ambipolar diffusion often dissipates the currents introduced by the emergence. We note that this does not always occur since it depends on the magnetic field configuration as detailed in Section 4.2.

One of the most exciting aspects of the GOL simulation is that it is the first Bifrost simulation in which jets that resemble type II spicules are ubiquitously formed. These jets are formed by the release of magnetic tension through a complex set of processes in which ambipolar diffusion plays a key role. The resulting jets show very high speeds, heights, and thermal evolution that are similar to those observed in type II spicules. The simulated jets occur mostly in the vicinity of the strongfield regions, similar to what is observed on the Sun. Ambipolar diffusion is required for the formation of these violent jets: it allows fields to diffuse into the chromosphere, concentrates electrical currents into narrow regions that enhance the magnetic tension, and leads to the dissipation of currents and subsequent heating of spicular plasma during the later phases of the evolution. Our modeled spicules thus nicely reproduce the observational indications for heating from chromospheric to TR temperatures during the spicular lifetime (Pereira et al. 2014; Skogsrud et al. 2015). This spicule formation process is further detailed in Martiunez-Sykora et al. (2017a). The differences in spicule properties between the GOL and non-GOL models play an important role in explaining the different thermodynamic stratifications we have found in these models.

Our simulations also show evidence of dynamics and mass loading that is directly caused by reconnection (unlike the above-mentioned spicule formation). For example, we find interesting dynamics in regions with almost horizontal field lines above intergranular lanes where reconnection with neighboring cold bubbles leads to U-shaped magnetic field lines. This type of reconnection does not produce jets that penetrate into the corona, but it does help to fill the upper chromosphere with plasma. It is tempting to speculate that this process may play a role in providing mass to prominences. The role of the ambipolar diffusion in this process is to (1) diffuse the magnetic field narrowing the currents at the location of the reconnection, (2) lift more plasma into the upper chromosphere, and (3) heat the cooler regions of the chromosphere.

Ambipolar diffusion also has another surprising effect: we find that the magnetic field is sometimes decoupled from the thermodynamic structures. This occurs where ambipolar diffusion is large, the electrical current is perpendicular to the magnetic field lines, and the thermodynamic timescales are of the same order as the ambipolar timescales (see MartínezSykora et al. 2016, for details). This has (at least) three interesting consequences.

First, our GOL simulation shows that the field line connectivity often changes during the evolution of spicules. This becomes particularly apparent toward the end of the spicule lifetime. This may provide an explanation to observations that suggest that chromospheric fibrils do not necessarily follow the magnetic field direction (de la Cruz Rodríguez \& Socas-Navarro 2011).

Another consequence is that it may invalidate modeling approaches that are based on tracking the thermodynamic evolution along "flux tubes," i.e., 1D hydrodynamic models. Clearly such models cannot properly capture the thermodynamic evolution of plasma in regions where ambipolar diffusion decouples the plasma from the magnetic field.

And finally, such decoupling may also affect magnetic field extrapolation methods that incorporate the direction of chromospheric features to constrain the extrapolation from photospheric magnetograms. Clearly such methods assume that chromospheric features are aligned with the magnetic field direction. Our simulation shows that this assumption breaks down when the ambipolar diffusion is significant (see 
Martínez-Sykora et al. 2016, for details). More generally, another challenge for such extrapolation methods is the fact that the ambipolar diffusion in the chromosphere shows strong variations, so that regions with strong ambipolar diffusion may be more potential, while at the boundaries between regions of strong and weak ambipolar diffusion the magnetic field lines may undergo very significant changes in the magnetic connectivity.

While these simulations show exciting results and potentially significantly reduce the known discrepancies between Bifrost models and observations of the chromosphere, several disclaimers are important to note. While the presence of features that resemble type II spicules in the GOL simulation may well reduce or even resolve long-standing issues with chromospheric simulations, a proper comparison with synthetic observables and an expansion of these results into three dimensions is required to settle this issue. This is a major effort that will be the subject of a follow-up paper. Another issue that needs to be addressed in the future is the fact that the current GOL simulation does not include time-dependent hydrogen or helium ionization, both of which will likely impact the values and spatiotemporal distribution of the ambipolar diffusion. We would expect that the ambipolar diffusion gradients are reduced owing to the time-dependent hydrogen and helium ionization (Leenaarts et al. 2007; Golding et al. 2014). In order to quantify the differences, future work needs to be based on simulations that take into account time-dependent hydrogen and helium ionization. Finally, the GOL is valid as long as the timescales are much larger than the ion-neutral collision frequencies. It is not clear whether this condition is always fulfilled close to and in the TR. If this condition is not fulfilled, it may lead to drift between ions and neutrals (Martínez-Sykora et al. 2012), which requires a multifluid code to properly treat. This could alter some of the results shown here.

We gratefully acknowledge support by NASA grants NNX11AN98G, NNM12AB40P, NNX16AG90G, and NNH15ZDA001N-HSR and NASA contracts NNM07AA01C (Hinode) and NNG09FA40C (IRIS). This research was supported by the European Research Council under the European Union's Seventh Framework Programme (FP7/ 2007-2013)/ERC Grant agreement no. 291058. The simulations have been run on clusters from the Notur project and the Pleiades cluster through the computing project s1061 from the High End Computing (HEC) division of NASA. We thankfully acknowledge the support of the Research Council of Norway through grant 230938/F50 and through grants of computing time from the Programme for Supercomputing. This work has benefited from discussions at the International Space Science Institute (ISSI) meetings on "Heating of the Magnetized Chromosphere," where many aspects of this paper were discussed with other colleagues. To analyze the data we have used IDL. We are very grateful to J. Vranjes for providing us the cross section tables, as well as interesting conversations on subjects related to this paper. Finally, we want to thank the referee for the help with improving the manuscript.

\section{Appendix \\ Data Access}

The full numerical domain with all the variables (listed in Table 2 in Carlsson et al. 2016) of the GOL simulation is freely available at the http://iris.lmsal.com/modeling.html Web page, similar to the previously published enhanced network simulation (Carlsson et al. 2016). For the current simulation, we also include the ambipolar diffusion, Joule heating coming from the ambipolar diffusion, and electric current variables $\left(\eta_{\mathrm{amb}}, q_{j \mathrm{amb}}\right.$, and $i x, i y, i z$, respectively).

The current simulation has a different magnetic field configuration than the previously published enhanced network simulation. Since the magnetic field is the main free parameter of these realistic simulations, our current simulation thus complements the Carlsson et al. (2016) simulation. In addition, the current simulation is only $2.5 \mathrm{D}$ but includes partial ionization effects. It does not include nonequilibrium ionization, but the domain is larger and with greater resolution. While using these models, one has to take into account their limitations (see below and Carlsson et al. 2016). In addition, one has to take into account that due to fast chromospheric expansion experienced in the GOL simulation (leading to cooler voids in the chromosphere), we had to increase from 1600 to $1800 \mathrm{~K}$ the ad hoc heating at $t=1460 \mathrm{~s}$ $($ snapshot $=352)$.

Each snapshot is separated by roughly $10 \mathrm{~s}$ and starts with $t=0 \mathrm{~s}$ at the snapshot number 200. The first available snapshot is at 280 , which is $800 \mathrm{~s}$ after the partial ionization effects are switched on. The snapshots that are available cover the snapshots between 280 and 370, i.e., $\sim 13.8$ minutes. This time period includes the full lifetime of several type II spicules including their impact on the corona (De Pontieu et al. 2017; for details on this topic, see Martiunez-Sykora et al. 2017a).

Following the same format as for the Bifrost simulation that was previously made available (Carlsson et al. 2016), the data are in FITS files with 2D cubes $(x, z)$ with one variable for each file. The $x$-axis is equidistant and can be generated using FITS keywords, while the $z$-grid is nonuniform and is given in a FITS extension.

The file names are of the form BIFROST_en096014_gol_<var $>$ _ $<$ snap $>$.fits, where the annotation en096014_gol comes from "enhanced network," $96 \mathrm{Mm}$ and $14 \mathrm{~km}$ are the horizontal size and grid spacing, respectively, and "gol" represents that the simulation includes ion-neutral interaction effects, i.e., the GOL. Similar to the previously published simulation, <var $>$ is the variable name listed in the search Web page, and $<$ snap $>$ is the snapshot number.

All variables have been cell centered on a right-handed system with $\mathrm{z}$ increasing upward. Index runs the same way as the axis, which means that $z[1]$ is at the bottom and $z[n z]$ at the top.

All units are SI and given in FITS keywords (Mm, $\mathrm{m} \mathrm{s}^{-1}, \mathrm{~kg}$ $\mathrm{m} \mathrm{s}^{-1}, \mathrm{~T}, \mathrm{~W} \mathrm{~m}^{-3}, \mathrm{~nm}, \mathrm{~T}$, etc.).

Metadata are given in the FITS header. This data release is part of the IRIS project, and an explanation of the FITS keywords is given in IRIS Technical Note (ITN) 33. Software to analyze the simulation data is provided in SolarSoft (SSW/ IRIS) with descriptions in ITN 34. Synthetic observables will also be made publicly available (see ITN 35). Papers published based on the simulation presented here should cite both the code description paper (Gudiksen et al. 2011) and the current paper.

\section{ORCID iDs}

Juan Martínez-Sykora (iD https://orcid.org/0000-00020333-5717

Bart De Pontieu (DD https://orcid.org/0000-0002-8370-952X 
Mats Carlsson (1) https://orcid.org/0000-0001-9218-3139

Viggo H. Hansteen (i) https://orcid.org/0000-0003-0975-6659

Daniel Nóbrega-Siverio (ib https://orcid.org/0000-00027788-6482

Boris V. Gudiksen (10) https://orcid.org/0000-0003-0547-4902

\section{References}

Abbett, W. P. 2007, ApJ, 665, 1469

Acheson, D. J. 1979, SoPh, 62, 23

Arber, T. D., Botha, G. J. J., \& Brady, C. S. 2009, ApJ, 705, 1183

Arber, T. D., Haynes, M., \& Leake, J. E. 2007, ApJ, 666, 541

Archontis, A., Moreno-Insertis, F., Galsgaard, K., Hood, A., \& O'Shea, E. 2004, A\&A, 426, 1047

Asensio Ramos, A., de la Cruz Rodríguez, J., Martínez González, M. J., \& Socas-Navarro, H. 2017, A\&A, 599, A133

Athay, R. G., \& Holzer, T. E. 1982, ApJ, 255, 743

Bingert, S., \& Peter, H. 2011, A\&A, 530, A112

Braginskii, S. I. 1965, RvPP, 1, 205

Carlsson, M., Hansteen, V. H., de Pontieu, B., et al. 2007, PASJ, 59, 663

Carlsson, M., Hansteen, V. H., Gudiksen, B. V., Leenaarts, J., \& De Pontieu, B. 2016, A\&A, 585, A4

Carlsson, M., \& Leenaarts, J. 2012, A\&A, 539, A39

Carlsson, M., Leenaarts, J., \& De Pontieu, B. 2015, ApJL, 809, L30

Cheung, M. C. M., \& Cameron, R. H. 2012, ApJ, 750, 6

Courant, R., Friedrichs, K., \& Lewy, H. 1928, MatAn, 100, 32

Cowling, T. G. 1957, Magnetohydrodinamics (Bristol: Adam Hilger, Ltd.)

de la Cruz Rodríguez, J., \& Socas-Navarro, H. 2011, A\&A, 527, L8

De Pontieu, B. 1999, A\&A, 347, 696

De Pontieu, B., De Moortel, I., Martínez-Sykora, J., \& McIntosh, S. W. 2017, ApJL, submitted

De Pontieu, B., \& Haerendel, G. 1998, A\&A, 338, 729

De Pontieu, B., Martens, P. C. H., \& Hudson, H. S. 2001, ApJ, 558, 859

De Pontieu, B., McIntosh, S., Hansteen, V. H., et al. 2007a, PASJ, 59, S655

De Pontieu, B., McIntosh, S. W., Carlsson, M., et al. 2007b, Sci, 318, 1574

De Pontieu, B., McIntosh, S. W., Carlsson, M., et al. 2011, Sci, 331, 55

Dere, K. P., Bartoe, J.-D. F., \& Brueckner, G. E. 1989, SoPh, 123, 41

Díaz, A. J., Khomenko, E., \& Collados, M. 2014, A\&A, 564, A97

Fontenla, J. M., Avrett, E. H., \& Loeser, R. 1993, ApJ, 406, 319

Forteza, P., Oliver, R., Ballester, J. L., \& Khodachenko, M. L. 2007, A\&A, 461,731

Galsgaard, K., \& Nordlund, Å. 1995, A 3D MHD code for Parallel Computers (http://sirrah.troja.mff.cuni.cz/ toast/archiv/nordlund-95.ps)

Georgoulis, M. K., Rust, D. M., Bernasconi, P. N., \& Schmieder, B. 2002, ApJ, 575,506

Golding, T. P., Carlsson, M., \& Leenaarts, J. 2014, ApJ, 784, 30

Goodman, M. L., \& Judge, P. G. 2012, ApJ, 751, 75

Gudiksen, B. V., Carlsson, M., Hansteen, V. H., et al. 2011, A\&A, 531, A154

Gudiksen, B. V., \& Nordlund, А̊. 2002, ApJL, 572, L113

Gudiksen, B. V., \& Nordlund, ̊.. 2005, ApJ, 618, 1020

Hansteen, V., Guerreiro, N., De Pontieu, B., \& Carlsson, M. 2015, ApJ, 811, 106

Hansteen, V. H., De Pontieu, B., Rouppe van der Voort, L., van Noort, M., \& Carlsson, M. 2006, ApJL, 647, L73

Hansteen, V. H., Hara, H., De Pontieu, B., \& Carlsson, M. 2010, ApJ, 718, 1070

Hayek, W., Asplund, M., Carlsson, M., et al. 2010, A\&A, 517, A49

Heggland, L., De Pontieu, B., \& Hansteen, V. H. 2007, ApJ, 666, 1277

Heggland, L., Hansteen, V. H., De Pontieu, B., \& Carlsson, M. 2011, ApJ, 743,142
Huba, J. D. 2003, in Space Plasma Simulation, Vol. 615 ed. J. Büchner, C. Dum, \& M. Scholer (Berlin: Springer), 166

Iijima, H., \& Yokoyama, T. 2015, ApJL, 812, L30

Judge, P. G., de Pontieu, B., McIntosh, S. W., \& Olluri, K. 2012, ApJ, 746, 158

Khomenko, E., \& Collados, M. 2012, ApJ, 747, 87

Khomenko, E., Díaz, A., de Vicente, A., Collados, M., \& Luna, M. 2014 A\&A, 565, A45

Leake, J. E., \& Arber, T. D. 2006, A\&A, 450, 805

Leake, J. E., Arber, T. D., \& Khodachenko, M. L. 2005, A\&A, 442, 1091

Leake, J. E., DeVore, C. R., Thayer, J. P., et al. 2014, SSRv, 184, 107

Leake, J. E., \& Linton, M. G. 2013, ApJ, 764, 54

Leenaarts, J., Carlsson, M., Hansteen, V., \& Gudiksen, B. V. 2011, A\&A, 530, A124

Leenaarts, J., Carlsson, M., Hansteen, V., \& Rutten, R. J. 2007, A\&A, 473, 625

Madjarska, M. S., Vanninathan, K., \& Doyle, J. G. 2011, A\&A, 532, L1

Martínez-Sykora, J., De Pontieu, B., Carlsson, M., \& Hansteen, V. 2016, ApJL, 831, L1

Martínez-Sykora, J., De Pontieu, B., \& Hansteen, V. 2012, ApJ, 753, 161

Martínez-Sykora, J., De Pontieu, B., Hansteen, V., \& Carlsson, M. 2015, RSPTA, 373, 40268

Martínez-Sykora, J., De Pontieu, B., Hansteen, V., \& McIntosh, S. W. 2011, ApJ, 732, 84

Martínez-Sykora, J., De Pontieu, B., Leenaarts, J., et al. 2013, ApJ, 771, 66

Martínez-Sykora, J., Hansteen, V., \& Carlsson, M. 2008, ApJ, 679, 871

Martínez-Sykora, J., Hansteen, V., \& Carlsson, M. 2009a, ApJ, 702, 129

Martínez-Sykora, J., Hansteen, V., DePontieu, B., \& Carlsson, M. 2009b, ApJ, 701, 1569

Martiunez-Sykora, J., De Pontieu, B., Hansteen, V. H., et al. 2017a, Sci, 356,1269

McIntosh, S. W., de Pontieu, B., Carlsson, M., et al. 2011, Natur, 475, 477

Nóbrega-Siverio, D., Moreno-Insertis, F., \& Martínez-Sykora, J. 2016, ApJ, 822,18

Orozco Suárez, D., Asensio Ramos, A., \& Trujillo Bueno, J. 2015, ApJL, 803, L18

Ortiz, A., Bellot Rubio, L. R., Hansteen, V. H., de la Cruz Rodríguez, J., \& Rouppe van der Voort, L. 2014, ApJ, 781, 126

Osterbrock, D. E. 1961, ApJ, 134, 347

Pandey, B. P., \& Wardle, M. 2008, MNRAS, 385, 2269

Parker, E. N. 2007, Conversations on Electric and Magnetic Fields in the Cosmos (Princeton, NJ: Princeton Univ. Press)

Penn, M. J., Schad, T., \& Cox, E. 2011, ApJ, 734, 47

Pereira, T. M. D., De Pontieu, B., \& Carlsson, M. 2012, ApJ, 759, 18

Pereira, T. M. D., De Pontieu, B., Carlsson, M., et al. 2014, ApJL, 792, L15

Rempel, M. 2017, ApJ, 834, 10

Skogsrud, H., Rouppe van der Voort, L., De Pontieu, B., \& Pereira, T. M. D. 2015, ApJ, 806, 170

Soler, R., Andries, J., \& Goossens, M. 2012, A\&A, 537, A84

Soler, R., Ballester, J. L., \& Zaqarashvili, T. V. 2015, A\&A, 573, A79

Soler, R., Carbonell, M., \& Ballester, J. L. 2013, ApJS, 209, 16

Soler, R., Oliver, R., \& Ballester, J. L. 2009, ApJ, 699, 1553

Stein, R. F., \& Nordlund, A. 2006, ApJ, 642, 1246

Sterling, A. C., Harra, L. K., \& Moore, R. L. 2010, ApJ, 722, 1644

Suematsu, Y., Wang, H., \& Zirin, H. 1995, ApJ, 450, 411

Tomczyk, S., McIntosh, S. W., Keil, S. L., et al. 2007, Sci, 317, 1192

Tortosa-Andreu, A., \& Moreno-Insertis, F. 2009, A\&A, 507, 949

Tsiropoula, G., Tziotziou, K., Kontogiannis, I., et al. 2012, SSRv, 169, 181

von Steiger, R., \& Geiss, J. 1989, A\&A, 225, 222

Vranjes, J., \& Krstic, P. S. 2013, A\&A, 554, A22

Vranjes, J., Poedts, S., Pandey, B. P., \& de Pontieu, B. 2008, A\&A, 478, 553

Withbroe, G. L., \& Noyes, R. W. 1977, ARA\&A, 15, 363 